\title{
INTERPOLATION AND GLEASON PARTS IN $L$-DOMAINS
}

BY

\author{
MICHAEL FREDERICK BEHRENS
}

\begin{abstract}
We describe the closure of $[-1 / 2,0)$ in the maximal ideal space $\mathscr{M}(\mathscr{D})$ of $H^{\infty}(\mathscr{D})$ for an arbitrary $L$-domain $\mathscr{D}$. For $L$-domains satisfying $\sup \left(c_{n+1} / c_{n}\right)<1$ and $\sum\left(r_{n} / c_{n}\right)^{p}<\infty$, some $p \geqslant 1$, we describe all interpolation sequences for $H^{\infty}(\mathscr{D})$, we show that a homomorphism (except the distinguished homomorphism, when it exists) lies in a nontrivial Gleason part if and only if it is contained in the closure of an interpolating sequence, and we describe all the analytic structure occurring in $\mathscr{M}(\mathscr{D})$.
\end{abstract}

1. Introduction. Let $\mathscr{M}(\mathscr{D})$ be the maximal ideal space of the Banach algebra $H^{\infty}(\mathscr{D})$ of all bounded analytic functions on a planar domain $\mathscr{D}$, equipped with the supremum norm. In the case when $\mathscr{D}$ is an $L$-domain, $\mathscr{D}=(\Delta \backslash\{0\}) \backslash \cup \Delta_{n}$, where $\Delta$ is the open unit disk and $\left\{\Delta_{n}\right\}$ is a sequence of closed disks with radii $r_{n}$ and centers $c_{n}$ converging to 0 on the positive axis. $\mathscr{M}(\mathscr{D})$ was studied by Zalcman in [9]. A striking phenomenon in $L$-domains is that the limit of $f(r)$, as $r$ approaches 0 along the negative axis, exists for all $f \in H^{\infty}(\mathscr{D})$ if $\Sigma\left(r_{n} / c_{n}\right)<\infty$. This means that the closure of $\left[\frac{-1}{2}, 0\right)$ in $\mathscr{M}(\mathscr{D})$ is obtained by adjoining a single point, the "distinguished" homomorphism defined by $\Phi_{0}(f)=\lim _{r \uparrow 0} f(r)$. In $\$ 2$ we describe the closure of $\left[\frac{-1}{2}, 0\right)$ in $\mathscr{M}(\mathscr{D})$ for an arbitrary $L$-domain. In particular, we show that if $\Sigma\left(r_{n} / c_{n}\right)=\infty$, then the closure of $\left[\frac{-1}{2}, 0\right)$ in $\mathscr{M}(\mathscr{D})$ is homeomorphic to the closure of $[0,1)$ in $\mathscr{M}(\Delta)$, and that a sequence in $\left[\frac{-1}{2}, 0\right)$ is an $H^{\infty}(\mathscr{D})$ interpolation sequence if and only if it is separated in the Carathéodory metric on $\mathscr{D}$.

Major objects of interest in $\mathscr{M}(\mathscr{D})$ are the analytic sets, the Gleason parts, and the interpolating sequences. We will give a complete description of these aspects of $\mathscr{M}(\mathscr{D})$ for a class of $L$-domains. This generalizes [3] and is motivated by a study of the extension $\mathscr{D}^{*}$ of $\mathscr{D}$ given in Abraham Robinson's theory of nonstandard analysis $[1,2]$.

In [3] we studied $L$-domains $\mathscr{D}=(\Delta \backslash\{0\}) \backslash \cup \Delta_{n}$ which satisfied $\sup \left(c_{n+1} / c_{n}\right)<$ 1 and $\Sigma\left(r_{n} / c_{n}\right)<\infty$. We showed that, with the exception of the Gleason part containing the distinguished homomorphism, each nontrivial Gleason part in $\mathscr{M}(\mathscr{D})$ is an analytic disk, and the Gleason part containing $\Phi_{0}$ is equal to $\mathscr{D}$ plus a set homeomorphic to $(\beta N \backslash N) \times \Delta$, with $(\beta N \backslash N) \times\{0\}$ identified with $\Phi_{0}$.

In $\S 3$ we consider domains $\mathscr{D}=\mathscr{E} \backslash \cup \Delta_{n}$ obtained by excising from some domain $\mathscr{E}$ a sequence of closed disks $\Delta_{n}$ satisfying the following condition: there are disjoint

Received by the editors October 4, 1977 and, in revised form. October 3, 1983.

1980 Mathematics Subject Classification. Primary 30D40: Secondary 03H05.

(c) 1984 American Mathematical Society $0002-9947 / 84 \$ 1.00+\$ .25$ per page 
disks $D_{n}$ with the same centers as $\Delta_{n}$, contained in $\mathscr{E}$, with $\sum\left(\operatorname{rad} \Delta_{n} / \operatorname{rad} D_{n}\right)^{p}<\infty$, some $p \geqslant 1$.

When $p=1$, such a sequence of disks was said in [4] to be hyperbolically rare. We describe all interpolating sequences in $\mathscr{D}$ which are "near" $U \Delta_{n}$. When $\mathscr{D}$ is an $L$-domain satisfying $\sup \left(c_{n+1} / c_{n}\right)<1$ and $\Sigma\left(r_{n} / c_{n}\right)^{p}<\infty$, some $p \geqslant 1$, we are able to describe all $H^{\infty}(\mathscr{D})$ interpolating sequences. This is the only class of infinitely connected domains for which such a description is known.

In $\S 4$ we consider a particular subset $\mathscr{T}$ of $\mathscr{M}(\mathscr{D})$, for the domains $\mathscr{D}=\mathscr{E} \backslash \cup \Delta_{n}$ considered in $\$ 3$, defined by

$$
\mathscr{T}=\bigcap_{N, R} \overline{\bigcup_{N}^{\infty} S\left(c_{n}, R r_{n}\right) \cap \mathscr{D},}
$$

where $R>1$, the closure is in $\mathscr{M}(\mathscr{D})$, and $S(c, r)=\{z:|z-c|<r\}$ is the open disc with center $c$ and radius $r$. (We use this notation throughout the paper.) $\mathscr{T}$ is the class of homomorphisms in $\mathscr{M}(\mathscr{D})$ which are approached "tangentially" along $\cup \Delta_{n}$. Using the description of interpolating sequences in \$3, and generalizing Hoffman's factorization theorems from [7], we show that each nontrivial Gleason part in $\mathscr{T}$ is an analytic disk, and a point in $\mathscr{T}$ lies in a nontrivial Gleason part if and only if it is contained in the closure of an interpolating sequence.

In $\$ 5$ we return to the class of $L$-domains $\mathscr{D}=(\Delta \backslash\{0\}) \backslash \cup \Delta_{n}$, with $\sup \left(c_{n+1} / c_{n}\right)<1$ and $\Sigma\left(r_{n} / c_{n}\right)^{p}<\infty$, and give a complete description of the Gleason parts for such domains. The results of $\S 4$ show that, in this case, a Gleason part of $\mathscr{M}(\mathscr{D})$ which does not intersect the closure of $\left[\frac{-1}{2}, 0\right)$ in $\mathscr{M}(\mathscr{D})$ is either a point part or an analytic disk, and a point in $\mathscr{M}(\mathscr{D})$ whose Gleason part does not intersect the closure of $\left[\frac{-1}{2}, 0\right)$ in $\mathscr{H}(\mathscr{D})$ is contained in a nontrivial Gleason part if and only if it is contained in the closure of an interpolating sequence. When $p=1$, the first assertion was proved in [3], but the second is new, and the Gleason part containing $\Phi_{0}$ is as described in [3]. When $p>1$, no "distinguished" homomorphism exists. In this case, using the results of $\S 2$, we show that each Gleason part of $\mathscr{M}(\mathscr{D})$ (except $\mathscr{D})$ which intersects the closure of $\left[\frac{-1}{2}, 0\right)$ in $\mathscr{M}(\mathscr{D})$ is a bijective continuous image of the following kind of space: For each real number $r$, let $K_{r}$ be a compact subset of $\beta N \backslash N$. In the space $R \cup \cup_{r}\left(K_{r} \times \Delta\right)$ identify each set $\{r\} \cup\left(K_{r} \times\{0\}\right)$ with a point. The resulting space is a "hedge" and each Gelfand transform is analytic on each "petal" $\{\omega\} \times \Delta, \omega \in K_{r}$.

We conclude this paper with a brief discussion of certain ideas in Abraham Robinson's theory of nonstandard analysis. Behavior of the nonstandard extension of the set $\mathscr{D}$ is intimately connected with the existence of analytic sets in $\mathscr{M}(\mathscr{D})$.

2. Approach to zero along the negative axis in $L$-domains. First some notation and definitions. On a planar domain $\mathscr{D}$ the Carathéodory metric $\pi$ is defined by

$$
\pi(z, w)=\sup \left\{\psi_{0}(f(z), f(w)): f \in H^{\infty}(\mathscr{D}),\|f\| \leqslant 1\right\},
$$

where $\psi_{0}$ is the Poincaré metric on the open unit disk $\Delta$. Any analytic function decreases distance in the Carathéodory metric: If $f$ maps $\left(\mathscr{D}_{1}, \pi_{1}\right)$ into $\left(\mathscr{D}_{2}, \pi_{2}\right)$ and is analytic, then $\pi_{2}(f(z), f(w)) \leqslant \pi_{1}(z, w)$ for all $z, w \in \mathscr{D}_{1}$. A sequence $\left\{\alpha_{n}\right\} \subset \mathscr{D}$ 
is said to be $\pi$-separated if $\inf _{n \neq m} \pi\left(\alpha_{n}, \alpha_{m}\right)>0$. For example, an interpolating sequence $\left\{\alpha_{n}\right\}$ in $\mathscr{D}$ is $\pi$-separated since there is a constant $M$ and functions $f_{n} \in H^{\infty}(\mathscr{D})$ with $\left\|f_{n}\right\| \leqslant M$ and $f_{n}\left(\alpha_{m}\right)=\delta_{n, m}$, and hence inf ${ }_{n \neq m} \pi\left(\alpha_{n}, \alpha_{m}\right) \geqslant 1 / M$. The $\pi$-distance between $A$ and $B$ is defined to be

$$
\pi(A, B)=\inf \{\pi(a, b): a \in A, b \in B\}
$$

and $A$ and $B$ are said to be $\pi$-separated from each other if $\pi(A, B)>0$.

For a domain $\mathscr{D} \subset \mathbf{C}$, the Gleason part containing $m \in \mathscr{M}(\mathscr{D})$ will be denoted by $\mathscr{G}(m)$. The part metric on $\mathscr{M}(\mathscr{D})$ is given by

$$
p(m, n)=\sup \left\{|\hat{f}(m)|: f \in H^{\infty}(\mathscr{D}),\|f\| \leqslant 1, \hat{f}(n)=0\right\} .
$$

A metric $\pi$ is defined on $\mathscr{G}(m)$ by $\pi=\log [(1+p) /(1-p)]$. This definition agrees with the previous one on $\mathscr{D}$ and satisfies the following: If $\left\{z_{\lambda}\right\}$ and $\left\{w_{\lambda}\right\}$ are nets in $\mathscr{D}$ with $\lim z_{\lambda}=m$ and $\lim w_{\lambda}=n$, where $m$ and $n$ are in the same Gleason part in $\mathscr{M}(\mathscr{D})$, then $\pi(m, n) \leqslant \lim \sup \pi\left(z_{\lambda}, w_{\lambda}\right)$.

In this section $\mathscr{E}$ will always denote the horizontal strip $\mathscr{E}=\{z \in \mathrm{C}$ : $|\operatorname{Im} z|<4\}$. In $\Delta$ and $\mathscr{E}$, the Carathéodory and Poincaré metrics are equal. The advantage of considering $\mathscr{E}$ is that the real line plays a special role in $\mathscr{M}(\mathscr{E})$. The following statements are well known [6,7] and are stated here for easy reference in notation consistent with our usage.

$2.1 \quad$ (1) $\pi=d / 4$ on $R$, where $d$ is the euclidean metric on $R$.

(2) A sequence contained in $R$ is $\pi$-separated if and only if it is an $H^{\infty}(\mathscr{E})$ interpolating sequence.

(3) The closure of $R$ in $H^{\infty}(\mathscr{E})$ is homeomorphic to the maximal ideal space of the Banach algebra of all bounded uniformly continuous complex valued functions on $(R, d)$ equipped with the supremum norm.

(4) Every point of the closure of $R$ in $\mathscr{M}(\mathscr{E})$ lies in a nontrivial Gleason part.

(5) Let $\mathscr{F}$ be the closure in $\mathscr{M}(\mathscr{E})^{\mathscr{E}}$, equipped with the compact-open topology, of the set of mappings defined by $z \rightarrow z+r, r \in R$. Every element of $\mathscr{F}$ is a bijective analytic mapping of $\mathscr{E}$ onto a Gleason part of $\mathscr{M}(\mathscr{E})$ and the images of $\mathscr{E}$ under $\mathscr{F}$ cover the closure of $R$ in $\mathscr{M}(\mathscr{E})$.

(6) If $A, B \subset R$ are $\pi$-separated from each other in $\mathscr{E}$, then $A^{-\mathscr{M}(\mathscr{E})} \cap B^{-\mathscr{M}(\mathscr{E})}=\varnothing$.

(7) If $m \in A^{-M(\mathscr{E})}$ and $n \in B^{-M(\mathscr{E})}$ with $\pi(m, n)=M<\infty$, then $\pi(A, B) \leqslant M$.

(8) Let $m, n \in R^{-M(\mathscr{)})}$. Then $m$ and $n$ are in the same Gleason part and $\pi(m, n)=M<\infty$ if and only if there are nets $\left\{x_{\lambda}\right\},\left\{y_{\lambda}\right\}$ in $R$ with $\lim x_{\lambda}=m, \lim y_{\lambda}=n$ and $\lim \pi\left(x_{\lambda}, y_{\lambda}\right)=M$.

For an arbitrary $L$-domain $\mathscr{D}=(\Delta \backslash\{0\}) \backslash \cup \Delta_{n}$, let $E=R \cap \cup \Delta_{n}$. Define a function on $\mathscr{D}$ by $f_{E}(z)=\int_{E} d x /(x-z)$ and let $g$ be the restriction of $f_{E}$ to $\left[\frac{-1}{2}, 0\right)$. 
The following properties of $f_{E}$ and $g$ are elementary and easy to check:

2.2

(1) $f_{E}$ is analytic on $\mathscr{D}$.

(2) $\quad|\operatorname{Im} f|<4$ on $\mathscr{D}$.

(3) $g$ is strictly increasing.

(4) $g$ is bounded if and only if $\Sigma\left(r_{n} / c_{n}\right)<\infty$.

Since $f_{E}$ maps $\mathscr{D}$ into $\mathscr{E}$, it decreases the Carathéodory distance in these two domains. In particular, the restriction mapping $g:\left(\left[\frac{-1}{2}, 0\right), \pi\right) \rightarrow\left(\left[f\left(\frac{-1}{2}\right), a\right) d\right)$ is uniformly continuous, where $a=\lim _{r \uparrow 0} f_{E}(r)$. One direction in the following theorem is thus evident.

THEOREM 2.3. The mapping $g:\left(\left[\frac{-1}{2}, 0\right), \pi\right) \rightarrow\left(\left[g\left(\frac{-1}{2}\right), a\right), d\right)$ is uniformly bicontinuous.

Proof. Let $u<v<0$ with

$$
\int_{E} \frac{v-u}{(x-u)(x-v)} d x=\left|\int_{E} \frac{d x}{x-u}-\int_{E} \frac{d x}{x-v}\right|<\delta .
$$

Let $0<\varepsilon<1$ and suppose

$$
E \cap[\varepsilon|v|,|u| / \varepsilon] \text { contains an interval of the form }[r / 2, r] .
$$

Then

$$
\frac{(v-u)(r / 2)}{(r-u)(r-v)} \leqslant \int_{r / 2}^{r} \frac{(v-u) d x}{(x-u)(x-v)} \leqslant \int_{E} \frac{(v-u) d x}{(x-u)(x-v)}<\delta
$$

so $v-u \leqslant 2 \delta(r-u)(r-v) / r$ and, hence, $(v-u) /|u| \leqslant 4 \delta / \varepsilon$. If $\|f\| \leqslant 1, f \in$ $H^{\infty}(\mathscr{D})$ and $f(u)=0$, then by Schwarz's lemma for $\{z \in \mathbf{C}:|z-u|<|u|\}$, we have $|f(v)| \leqslant 4 \delta / \varepsilon$. Hence

$$
\pi(u, v) \leqslant \log \frac{1+4 \delta / \varepsilon}{1-4 \delta / \varepsilon} .
$$

If (*) is false, then $r_{n} / c_{n} \leqslant 1 / 2$ for $\varepsilon|v| \leqslant c_{n} \leqslant|u| / \varepsilon$, and there are circles $\gamma_{1}=\{z$ : $\left.|z|=r_{1}\right\}$ and $\gamma_{2}=\left\{z:|z|=r_{2}\right\}$ with $\gamma_{1}, \gamma_{2} \subset \mathscr{D}, \varepsilon|v| \leqslant r_{1} \leqslant 2 \varepsilon|v|$ and $|u| / 2 \varepsilon \leqslant r_{2} \leqslant$ $|u| / \varepsilon$. Let $A=\mathscr{D} \cap\left\{z: r_{1}<|z|<r_{2}\right\}$ and $\partial A=\gamma_{1} \cup \gamma_{2} \cup \cup_{n_{0}}^{n_{1}} \partial \Delta_{n}$. Let $f \in H^{\infty}(\mathscr{D})$ with $\|f\| \leqslant 1$. Then for $z \in A$,

$$
f(z)=\frac{1}{2 \pi i} \int_{\gamma_{2}} \frac{f(\xi) d \xi}{\xi-z}-\frac{1}{2 \pi i} \int_{\gamma_{1}} \frac{f(\xi) d \xi}{\xi-z}-\sum_{n_{1}}^{n_{0}} \frac{1}{2 \pi i} \int_{\gamma_{n}} \frac{f(\xi) d \xi}{\xi-z} .
$$

Now

$$
\begin{gathered}
\left|f_{1}(u)-f_{1}(v)\right| \leqslant\left|\frac{1}{2 \pi i} \int_{\gamma_{1}} \frac{f(\xi) d \xi}{\xi-u}\right|+\left|\frac{1}{2 \pi i} \int_{\gamma_{1}} \frac{f(\xi) d \xi}{\xi-v}\right| \leqslant 4 \varepsilon \\
\left|f_{2}(u)-f_{2}(v)\right|=\left|\frac{1}{2 \pi i} \int_{\gamma_{2}} \frac{(v-u) f(\xi) d \xi}{(\xi-u)(\xi-v)}\right| \leqslant 16 \varepsilon
\end{gathered}
$$


and

$$
\begin{aligned}
\left|f_{3}(u)-f_{3}(v)\right| & =\left|\frac{1}{2 \pi i} \int_{\cup_{n_{0}}^{n_{1}} \partial \Delta_{n}} \frac{(v-u) f(\xi) d \xi}{(\xi-u)(\xi-v)}\right| \\
& \leqslant \frac{1}{2 \pi} \int_{\cup_{n_{0}}^{n_{1}} \partial \Delta_{n}} \frac{(v-u)|d \xi|}{|\xi-u||\xi-v|} \\
& \leqslant \int_{R \cap \cup_{n_{0}}^{n_{1} \Delta_{n}}} \frac{(v-u) d x}{(x-u)(x-v)} \\
& \leqslant \int_{E} \frac{(v-u) d x}{(x-v)(x-u)} \leqslant \delta .
\end{aligned}
$$

COROllaRy 2.4. A sequence $\left\{\alpha_{n}\right\} \subset\left[\frac{-1}{2}, 0\right)$ is $\pi$-separated in $\mathscr{D}$ if and only if $\left\{g\left(\alpha_{n}\right)\right\}$ is $\pi$-separated in $\mathscr{E}$.

Sets $A, B \subset\left[\frac{-1}{2}, 0\right)$ are $\pi$-separated from each other if and only if $g(A)$ and $g(B)$ are $\pi$-separated from each other in $\mathscr{E}$.

If $\left\{g\left(\alpha_{n}\right)\right\}$ is interpolating for $H^{\propto}(\mathscr{E})$, then $H^{\infty}(\mathscr{E}) \circ g$ interpolates all bounded values on $\left\{\alpha_{n}\right\}$. Since a sequence in $R$ is interpolating for $H^{\infty}(\mathscr{E})$ if and only if it is $\pi$-separated in $\mathscr{E}$, and since an interpolating sequence in $\mathscr{D}$ is $\pi$-separated in $\mathscr{D}$, the following corollary is evident.

COROLlaRY 2.5. A sequence $\left\{\alpha_{n}\right\} \subset\left[\frac{-1}{2}, 0\right)$ is interpolating for $H^{x}(\mathscr{D})$ if and only if $\left\{g\left(\alpha_{n}\right)\right\}$ is interpolating for $H^{\infty}(\mathscr{E})$.

If $\left\{\alpha_{n}\right\} \subset\left[\frac{-1}{2}, 0\right)$ is $\pi$-separated, then $\left\{g\left(\alpha_{n}\right)\right\}$ is $\pi$-separated and hence interpolating for $H^{\infty}(\mathscr{E})$, so $\left\{\alpha_{n}\right\}$ is interpolating for $H^{\infty}(\mathscr{D})$.

COROLlaRY 2.6. A sequence $\left\{\alpha_{n}\right\} \subset\left[\frac{-1}{2}, 0\right)$ is interpolating for $H^{x}(\mathscr{D})$ if and only if $\left\{\alpha_{n}\right\}$ is $\pi$-separated in $\mathscr{D}$.

Theorem 2.3 says that $\pi(x, y)$ is small if and only if $\pi(g(x), g(y))$ is small. Another corollary to Theorem 3 is that $\pi(x, y)$ is large if and only if $\pi(g(x), g(y))$ is large. Since $\pi(g(x), g(y)) \leqslant \pi(x, y)$, one direction is trivial.

COROLlaRY 2.7. There is a $\delta>0$ such that $d(g(x), g(y)) \geqslant \delta \pi(x, y)$ whenever $\pi(x, y) \geqslant 1$.

Proof. There is a $\delta$ such that $d(g(x), g(y)) \leqslant \delta \Rightarrow \pi(x, y)<1 / 2$. Suppose $\delta N \leqslant d(g(x), g(y))<\delta(N+1)$. Let $g(x)=r_{0}<r_{1}<\cdots r_{N+1}=g(y)$ with $\mid x_{k}-$ $x_{k-1} \mid \leqslant \delta$. Then

$$
\pi(x, y) \leqslant \sum_{0}^{N} \pi\left(g^{-1}\left(r_{k+1}\right), g^{-1}\left(r_{k}\right)\right) \leqslant \frac{N+1}{2} \leqslant \frac{d(g(x), g(y))}{\delta} .
$$

The function $f_{E}$ has a unique continuous extension to a mapping $\tilde{f}_{E}$ of $\mathscr{M}(\mathscr{D})$ into $\mathscr{M}(\mathscr{E})$, defined by

$$
\tilde{f}_{E}(m)(h)=m\left(h \circ f_{E}\right), \quad m \in \mathscr{M}(\mathscr{D}), h \in H^{x}(\mathscr{E}) .
$$

Let $\tilde{g}$ be the restriction of $\tilde{f}_{E}$ to the closure of $\left[\frac{-1}{2}, 0\right)$ in $\mathscr{M}(\mathscr{D})$. 
THEOREM 2.8. The mapping $\tilde{g}$ is a homeomorphism of $\left[\frac{-1}{2}, 0\right)^{-\mathscr{M}(D)}$ onto $\left[f\left(\frac{-1}{2}\right), a\right)^{-M(\infty)}$.

Proof. It suffices to show that $\tilde{g}$ is one-to-one. Let $m, n \in\left[\frac{-1}{2}, 0\right)^{-M(D)} \backslash\left[\frac{-1}{2}, 0\right)$ with $m \neq n$. Let $f \in H^{\infty}(\mathscr{D})$ with $m(f)=0, n(f)=1$. Let

$$
A=\left\{x \in\left[\frac{-1}{2}, 0\right):|f(x)|<\frac{1}{3}\right\}, \quad B=\left\{x \in\left[\frac{-1}{2}, 0\right):|f(x)| \geqslant \frac{2}{3}\right\} .
$$

Then $m \in A^{-\mathscr{M}(\mathscr{D})}, n \in B^{-\mathscr{M}(\mathscr{D})}$, and $A$ and $B$ are $\pi$-separated from each other. Hence $\tilde{g}(m) \in g(A)^{-M(\mathscr{E})}, \tilde{g}(n) \in g(B)^{-\mathscr{N}(\mathscr{E})}$ and $g(A), g(B)$ are $\pi$-separated from each other. Thus $g(A)^{-\mathscr{M}(\mathscr{E})} \cap g(B)^{-\mathscr{M}(\mathscr{E})}=\varnothing$ and $\tilde{g}(m) \neq \tilde{g}(n)$.

The mapping $\tilde{g}$ also preserves Gleason parts. Let $\mathscr{G}(m)$ be the Gleason part in $\mathscr{M}(\mathscr{D})$ of $m \in\left[\frac{-1}{2}, 0\right)^{-\mathscr{M}(\mathscr{D})}$ and $\mathscr{G}(\tilde{g}(m))$ the Gleason part of $\tilde{g}(m)$ in $\mathscr{M}(\mathscr{E})$.

THEOREM 2.9. Let $m \in\left[\frac{-1}{2}, 0\right)^{-\mathscr{M}(\mathscr{D})}$. Then

$$
\tilde{g}\left(\mathscr{G}(m) \cap\left[\frac{-1}{2}, 0\right)^{-\mathscr{M}(\mathscr{D})}\right)=\left[f\left(\frac{-1}{2}\right), a\right)^{-\mathscr{M}(\mathscr{E})} \cap \mathscr{G}(\tilde{g}(m))
$$

and

$$
\tilde{g}:\left(\mathscr{G}(m) \cap\left[\frac{-1}{2}, 0\right)^{-\mathscr{M}(\mathscr{D})}, \pi\right) \rightarrow\left(\left[f\left(\frac{-1}{2}\right), a\right)^{-\mathscr{M}(\mathscr{E})} \cap \mathscr{G}(\tilde{g}(m)), \pi\right)
$$

is uniformly bicontinuous.

Proof. Clearly,

$$
\tilde{g}\left(\mathscr{G}(m) \cap\left[\frac{-1}{2}, 0\right)^{-\mathscr{M}(\mathscr{D})}\right) \subset\left[f\left(\frac{-1}{2}\right), a\right)^{-\mathscr{M}(\mathscr{E})} \cap \mathscr{G}(\tilde{g}(m)) .
$$

Conversely, we must show that $n \in\left[\frac{-1}{2}, 0\right)^{-\mathscr{M}(\mathscr{D})}, \tilde{g}(n) \in \mathscr{G}(\tilde{g}(m)) \Rightarrow n \in \mathscr{G}(m)$. Suppose $n \notin \mathscr{G}(m)$. Let $\varepsilon=e^{-(2 M / \delta)}$, where $M=\pi(\tilde{g}(m), \tilde{g}(n))$. There is an $f \in$ $H^{x}(\mathscr{D})$ with $\|f\| \leqslant 1, \hat{f}(m)=0$ and $|\hat{f}(n)|>1-\varepsilon$. Let

$$
C=\left\{x \in\left[\frac{-1}{2}, 0\right):|f(x)| \leqslant \varepsilon\right\} \text { and } D=\left\{x \in\left[\frac{-1}{2}, 0\right):|f(x)| \geqslant 1-\varepsilon\right\} .
$$

Then $m \in C^{-M(D)}$ and $\pi(C, D) \geqslant \log 1 / \varepsilon=2 M / \delta$. If $\delta$ is chosen as in Corollary 2.7, then $\pi(g(C),(D)) \geqslant 2 M$. Since $\tilde{g}(m) \in g(C)^{-\mathscr{M}(\mathscr{E})}$ and $\tilde{g}(n) \in g(D)^{-\mathscr{M}(\mathscr{E})}$, this is a contradiction.

The mapping $\tilde{f}_{E}$ decreases the $\pi$-metric. To see this we show that $\tilde{f}_{E}$ decreases the part metric $p$. If $m, n \in \mathscr{M}(\mathscr{D})$ then $p\left(\tilde{f}_{E}(m), \tilde{f}_{E}(n)\right)$ is equal to the supremum of $\left|\hat{h}\left(\tilde{f}_{E}(n)\right)\right|$ with $h \in H^{\infty}(E),\|h\| \leqslant 1$, and $\hat{h}\left(\tilde{f}_{E}(m)\right)=0$. Since $h \circ f_{E} \in H^{\infty}(\mathscr{D})$, $\left\|h \circ f_{E}\right\| \leqslant 1$, and $h \circ f_{E}(m)=\hat{h}\left(\tilde{f}_{E}(m)\right)=0$, it must be that

$$
\left|\hat{h}\left(\tilde{f}_{E}(n)\right)\right|=\left|h \circ f_{E}(n)\right| \leqslant p(m, n) .
$$

The mapping $\tilde{g}$ is thus uniformly continuous. To see that $\tilde{g}$ is uniformly continuous in the opposite direction, let $\tilde{g}\left(n_{0}\right), \tilde{g}\left(n_{1}\right) \in \mathscr{G}(\tilde{g}(m)) \cap\left[f\left(\frac{-1}{2}\right), a\right)^{-\mathscr{M}(\mathscr{E})}$ with $\pi\left(\tilde{g}\left(n_{0}\right), \tilde{g}\left(n_{1}\right)\right)<\delta$, where $\delta$ is chosen by Theorem 1 such that $\pi(g(x), g(y))<\delta$ $\Rightarrow \pi(x, y)<\varepsilon$. Then there are nets $\left\{x_{\lambda}\right\},\left\{y_{\lambda}\right\} \subset \mathbf{R}$ with $\lim x_{\lambda}=\tilde{g}\left(n_{0}\right), \lim y_{\lambda}=$ $\tilde{g}\left(n_{1}\right)$ and $\lim \pi\left(x_{\lambda}, y_{\lambda}\right)=\pi\left(n_{0}, n_{1}\right)<\delta$. Then $\lim g^{-1}\left(x_{\lambda}\right)=n_{0}, \lim g^{-1}\left(x_{\lambda}\right)=n_{1}$ and

$$
\pi(m, n) \leqslant \limsup \pi\left(g^{-1}\left(x_{\lambda}\right), g^{-1}\left(y_{\lambda}\right)\right)<\varepsilon
$$


If $\Sigma\left(r_{n} / c_{n}\right)<\infty$, then

$$
\lim _{\substack{z \uparrow 0 \\ z \in(1 / 2,0)}} \int_{E} \frac{d x}{x-z}=a<\infty
$$

and $\left[\frac{-1}{2}, 0\right)^{-M(D)}$ is homeomorphic to $\left[f\left(\frac{-1}{2}\right), a\right]$, so $\left[\frac{-1}{2}, 0\right)^{-\mathscr{M}(\mathscr{D})} \backslash\left[\frac{-1}{2}, 0\right)$ consists of a single point $\Phi_{0}=\tilde{g}^{-1}(a)$ which is in the same Gleason part as $\mathscr{D}$ and satisfies

$$
\lim _{\substack{x \uparrow 0 \\ x \in[-1 / 2,0)}} \pi\left(x, \Phi_{0}\right)=0 \text {. }
$$

If $\sum\left(r_{n} / c_{n}\right)=\infty$, Theorem 2.8 says that $\left[\frac{-1}{2}, 0\right)^{-M(D)} \backslash\left[\frac{-1}{2}, 0\right)$ is large and Theorem 2.9 says that the intersection of each Gleason part with $\left[\frac{-1}{2}, 0\right)^{-M(D)}$ is large.

THEOREM 2.10. Let $\Sigma\left(r_{n} / c_{n}\right)=\infty$ and let $\mathscr{G}$ be a Gleason part of $\mathscr{M}(\mathscr{D})$ which intersects $\left[\frac{-1}{2}, 0\right)^{-M(D)} \backslash\left[\frac{-1}{2}, 0\right)$. Then $\mathscr{G} \cap\left[\frac{-1}{2}, 0\right)^{-M(D)}$ is the continuous one-to-one image of the real line $R$ under a mapping $T$ which is uniformly bicontinuous when $\mathscr{G} \cap$ $\left[\frac{-1}{2}, 0\right)^{-M(D)}$ is equipped with the $\pi$-metric.

Proof. Let $m \in\left[\frac{-1}{2}, 0\right)^{-\mathscr{M}(\mathscr{D})} \cap \mathscr{G}$. Let $W: \mathscr{E} \rightarrow \mathscr{M}(\mathscr{E})$ be a Wermer map in the closure of $\left\{z \rightarrow z+r: r \in R^{+}\right\}$in $\mathscr{M}(\mathscr{E})^{\mathscr{E}}$ with $W(0)=\tilde{g}(m)$. Then $\left(\tilde{g}^{-1} \circ W\right) \mid R$, the restriction of $\tilde{g}^{-1} \circ W$ to $R$, works.

COROLLARY 2.11. If $\Sigma\left(r_{n} / c_{n}\right)=\infty$, then each point of $\left[\frac{-1}{2}, 0\right)^{-\mathcal{M}(\mathscr{D})}$ is contained in a nontrivial Gleason part.

3. Interpolating sequences. The interpolating sequences for $H^{\infty}(\Delta)$ are the sequences for which

$$
\delta\left(\left\{\alpha_{n}\right\}\right)=\inf \prod_{n \neq m} \rho\left(\alpha_{n}, \alpha_{m}\right)>0 .
$$

In some cases a similar condition can be given for a sequence which lies close to a sequence of holes in an infinitely connected domain to be interpolating. We begin with a useful observation which says that interpolation is local, and then consider interpolation in the annulus.

LEMMA 3.1. Let $\mathscr{D}$ be a bounded domain. Let $K_{1}, \ldots, K_{n}$ be $n$ disjoint compact subsets of $\partial \mathscr{D}$. Let $S=S_{1} \cup \cdots \cup S_{n}$ be a discrete subset of $\mathscr{D}$ with the $S_{i}$ mutually disjoint and with $S_{i}$ accumulating only at points of $K_{i}$. Then $S$ is interpolating for $H^{\infty}(\mathscr{D})$ if and only if each $S_{i}$ is interpolating for $H^{\infty}(\mathscr{D})$.

Proof. Invoke the Badé-Curtis Theorem [6] with the help of the function $\hat{z}$.

Lemma 3.2. Let $0<a<b<1, A=\{z \in \mathbf{C}: a<|z|<1\}, B=\{z \in \mathbf{C}: b<|z|$ $<1\}$, and $\left\{\alpha_{n}\right\}=S$ a sequence in $B$. Suppose $S$ is an interpolating sequence for $H^{\infty}(A)$ with norm $M$, i.e. for each sequence $\left\{a_{n}\right\}$ with $\left|a_{n}\right| \leqslant 1$, there is an $f \in H^{\infty}(A)$ with $\|f\| \leqslant M$ and $f\left(\alpha_{n}\right)=a_{n}$. Then $S$ is interpolating for $H^{\infty}(\Delta)$ and $\delta(S) \geqslant b^{n} / 4 M$, where $n$ is such that

$$
\frac{2 \pi a M}{b-a}\left(\frac{a}{b}\right)^{n} \leqslant \frac{1}{10}
$$


Proof. Choose $n$ such that $(* *)$ holds. Let $\left|a_{k}\right| \leqslant 1$. Choose $f_{1} \in H^{\infty}(A)$ with $\left\|f_{1}\right\|_{A} \leqslant M / b^{n}$ and $f_{1}\left(\alpha_{k}\right)=a_{k} / \alpha_{k}^{n}$. Let $g_{1}(z)=z^{n} f_{1}(z)$ and write

$$
\begin{aligned}
g_{1}(z) & =\int_{|=|=1} g_{1}(\xi) \frac{d \xi}{\xi-z}-\int_{|z|=a} g_{1}(\xi) \frac{d \xi}{\xi-z} \\
& =g_{1}^{(1)}(z)-g_{1}^{(2)}(z) .
\end{aligned}
$$

In $B$,

$$
\left|g_{1}^{(2)}(z)\right| \leqslant \frac{2 \pi a M}{b-a}\left(\frac{a}{b}\right)^{n} \leqslant \frac{1}{10}
$$

Hence

$$
\left\|g_{1}^{(1)}\right\|_{\Delta} \leqslant M / b^{n}+1 / 10 \leqslant 2 M / b^{n} \text { and }\left|a_{k}-g_{1}^{(1)}\left(\alpha_{k}\right)\right|=\left|g_{1}^{(2)}\left(\alpha_{k}\right)\right| \leqslant 1 / 10 \text {. }
$$

Repeating this process, starting with a function $f_{2} \in H^{\infty}(A)$, with $\left\|f_{2}\right\|_{A} \leqslant M /\left(10 b^{n}\right)$ and $f_{2}\left(\alpha_{k}\right)=\left(a_{k}-g_{1}^{(1)}\left(\alpha_{k}\right)\right) / \alpha_{k}^{n}$, we obtain a function $g_{2}^{(1)}(z)$ with

$$
\left\|g_{2}^{(1)}\right\|_{\perp} \leqslant 2 M /\left(10 b^{n}\right) \text { and }\left|\left(a_{k}-g_{1}^{(1)}\left(\alpha_{k}\right)\right)-g_{2}^{(1)}\left(\alpha_{k}\right)\right| \leqslant 1 / 10^{2} \text {. }
$$

After $m$ repetitions, we have $g_{m}^{(1)} \in H^{\infty}(\Delta)$ with $\left\|g_{m}^{(1)}\right\|_{د} \leqslant 2 M /\left(10^{m-1} b^{n}\right)$ and

$$
\left|\left(a_{k}-g_{1}^{(1)}\left(\alpha_{k}\right)-g_{2}^{(1)}\left(\alpha_{k}\right)-\cdots-g_{m-1}^{(1)}\left(\alpha_{k}\right)\right)-g_{m}^{(1)}\left(\alpha_{k}\right)\right| \leqslant 1 / 10^{m} .
$$

Thus $\sum_{m} g_{m}^{(1)}=h \in H^{\infty}(\Delta), h\left(\alpha_{k}\right)=a_{k}$, and $\|h\| \leqslant 4 M / b^{n}$, and hence $\delta(S) \geqslant$ $1 /\|h\|=b^{n} / 4 M$.

Lemma 3.2 gives the following necessary condition for interpolation near a sequence of holes in a domain.

LEMMA 3.3. Let $\mathscr{D}=\mathscr{E} \backslash \cup \Delta_{n}, \mathscr{E}$ an arbitrary domain, with $\Delta_{n}$ closed disks with radius $r_{n}$ and centers $c_{n}$ contained in $\mathscr{E}$. Suppose $R>1$ is such that the sets $S\left(c_{n}, R r_{n}\right)$ are mutually disjoint and $\overline{S\left(c_{n}, R r_{n}\right)} \subset \mathscr{E}$, and let $S \subset \cup S\left(c_{n}, R r_{n}\right)$ be an interpolating sequence for $H^{\propto}(\mathscr{D})$. Let $T_{n}(z)=r_{n} /\left(z-c_{n}\right)$. Then inf $\delta\left(T_{n}\left(S \cap S\left(c_{n}, R r_{n}\right)\right)\right)>0$.

Proof. The sequence $T_{n}\left(S \cap\left(S\left(c_{n}, R r_{n}\right)\right)\right)$ is contained in $\{z \in \mathrm{C}: 1 / R<|z|<$ $1\}$, and it is an interpolation sequence for $H^{\infty}\{z \in \mathrm{C}: \varepsilon / R<|z|<1\}$ for some $0<\varepsilon<1, \varepsilon$ independent of $n$. Apply Lemma 3.2.

With more restrictions on the sequence of holes $\left\{\Delta_{n}\right\}$, it is possible to show that the condition of Lemma 3.3 is sufficient to guarantee that a sequence interpolates $H^{\infty}(\mathscr{D})$.

THEOREM 3.4. Let $\mathscr{D}=\mathscr{E} \backslash \cup \Delta_{n}$ with $\Delta_{n}=\overline{S\left(c_{n}, r_{n}\right)}$. Suppose there are mutually disjoint disks $D_{n}=\overline{S\left(c_{n}, R_{n}\right)}$ with $R_{n}>r_{n}, D_{n} \subset \mathscr{E}$, and $\Sigma\left(r_{n} / R_{n}\right)^{p}<\infty$, some $p \geqslant 1$. Let $1<R<R_{n}$ for all $n$, let $S$ be a sequence in $\mathscr{D}$ which is contained in US $\left(c_{n}, R r_{n}\right)$, and let $T_{n}=r_{n} /\left(z-c_{n}\right)$. Then $S$ is interpolating for $H^{\infty}(\mathscr{D})$ if and only if inf $\delta\left(T_{n}\left(S \cap D_{n}\right)\right)>0$.

Proof. Let $\delta=\inf \delta\left(T_{n}\left(S \cap D_{n}\right)\right)>0$. Let $\varepsilon>0$ be such that if $\left\{\beta_{n}\right\} \subset \Delta$ with $\inf \left(\beta_{n}\right) \geqslant 1 / R, \delta\left(\left\{\beta_{n}\right\}\right) \geqslant \delta$, and $\left|a_{n}\right| \leqslant \varepsilon$, then there is an $f \in H^{\infty}(\Delta)$ with $f(0)=0$, $\|f\| \leqslant 1$, and $f\left(\beta_{n}\right)=a_{n}$. Let $M$ be such that $\sum_{M}^{\infty}\left(r_{n} / R_{N}\right)^{p}<\varepsilon / 10$. We will show that $S \cap \cup_{M}^{\infty} S\left(c_{n}, R r_{n}\right)$ is interpolating. Theorem 3.4 then follows from Lemma 3.1. 
Let $\left|a_{k}\right|<\varepsilon^{p}$. Pick $f_{1}^{(n)} \in H^{\infty}(\Delta)$ such that $\left\|f_{1}^{(n)}\right\| \leqslant 1, f_{1}^{(n)}(0)=0$, and $f_{1}^{(n)}\left(T_{n}\left(\alpha_{k}\right)\right)=a_{k}^{1 / p}$ for all $\alpha_{k} \in S \cap S\left(c_{n}, R r_{n}\right)$. Then

$$
\sum_{M}^{\infty}\left(f_{1}^{(n)} \circ T_{n}\right)^{p}=f_{1} \in H^{\infty}(\mathscr{D})
$$

and

$$
\left.\mid f_{1}\left(\alpha_{k}\right)-a_{k}\right)|=| \sum_{\substack{m \neq n \\ m>M}}\left(f_{1}^{(m)}\left(T_{m}\left(\alpha_{k}\right)\right)\right)^{p} \mid \leqslant \sum_{M}^{\infty}\left(\frac{r_{n}}{R_{n}}\right)^{p}<\frac{\varepsilon}{10} .
$$

Let $a_{k}^{(1)}=a_{k}-f_{1}\left(\alpha_{k}\right)$ and $f_{2}^{(n)}\left(T_{n}\left(\alpha_{k}\right)\right)=\left(a_{k}^{(1)}\right)^{1 / p}$ with $f_{2}^{(n)} \in H^{\infty}(\Delta), f_{2}^{(n)}(0)=$ 0 , and $\left\|f_{2}^{(n)}\right\| \leqslant 1 / 10$. Then

$$
\sum_{M}^{\infty}\left(f_{2}^{(n)} \circ T_{n}\right)^{p}=f_{2} \in H^{x}(\mathscr{D})
$$

and

$$
\begin{aligned}
\left|a_{k}-\left(f_{1}\left(\alpha_{k}\right)+f_{2}\left(\alpha_{k}\right)\right)\right| & =\left|\sum_{\substack{m \neq n \\
m \geqslant M}}\left(f_{2}^{(m)}\left(T_{m}\left(\alpha_{k}\right)\right)\right)^{p}\right| \\
& \leqslant \frac{1}{10}\left(\sum_{M}^{\infty}\left(\frac{r_{n}}{R_{n}}\right)^{p}\right)<\frac{\varepsilon}{10^{2}} .
\end{aligned}
$$

Inductively define functions $f_{n} \in H^{\infty}(\mathscr{D})$ with $\left\|f_{n}\right\| \leqslant 1 / 10^{n-1}\left\|f_{1}\right\|$ and $\mid a_{k}-$ $\left(f_{1}\left(\alpha_{k}\right)+\cdots+f_{n}\left(\alpha_{k}\right)\right) \mid<\varepsilon / 10^{n}$. The function $f=\sum f_{n} \in H^{\infty}(\mathscr{D})$ interpolates the values $\left\{a_{n}\right\}$ at $\left\{\alpha_{n}\right\}, f\left(\alpha_{n}\right)=a_{n}$.

We will now consider $L$-domains $(\mathscr{D} \backslash\{0\}) \backslash \cup \Delta_{n}$ with $c_{n+1} / c_{n} \leqslant c<1$ and $\sum\left(r_{n} / c_{n}\right)^{p}<\infty$, some $p \geqslant 1$. The following technical lemma lists some useful properties of such $L$-domains.

LEMMA 3.5. Let $\mathscr{D}=(\Delta \backslash\{0\}) \backslash \cup \Delta_{n}$ with $c_{n+1} / c_{n} \leqslant c<1$ and $\Sigma\left(r_{n} / c_{n}\right)^{p}<\infty$. Then there are $D_{n}=S\left(c_{n}, R_{n}\right)$ such that:

(1) The $D_{n}$ are mutually disjoint.

(2) $\Sigma\left(r_{n} / R_{n}\right)^{p}<\infty$.

Given $\varepsilon>0$ there is an $R>1$ and a $\delta>0$ such that

(3) For $c_{n}<\delta, R r_{n} \leqslant R_{n}$ and the $\pi$-diameter of $D_{n} \backslash S\left(c_{n}, R r_{n}\right)$ is less than $\varepsilon$.

(4) If $|z| \leqslant \delta$ and $z \notin U S\left(c_{n}, R r_{n}\right)$, then $\pi(-|z|, z)<\varepsilon$.

Proof. The constant $R_{n}=c_{n} / 2(1-c)$ works for (1) and (2). Let $M$ be a real number such that $\gamma_{1}=\left\{z \in \mathbf{C}:|z|=c_{n} / M\right\}$ and $\gamma_{2}=\left\{z \in \mathbf{C}:|z|=M c_{n}\right\}$ are contained in $\mathscr{D}$. For $c_{n} / M<|z|<M c_{n}, z \in \mathscr{D}$ and $f \in H^{\infty}(\mathscr{D})$, Cauchy's theorem gives

$$
f(z)=\int_{\gamma_{2}} \frac{f(\xi) d \xi}{\xi-z}-\sum\left\{\int_{\partial \Delta_{m}} \frac{f(\xi) d \xi}{\xi-z}: \frac{c_{n}}{M}<c_{m}<M c_{n}\right\}-\int_{\gamma_{1}} \frac{f(\xi) d \xi}{\xi-z} .
$$

If $M$ is sufficiently large, then the first and last integral will each have variation less than $\varepsilon\|f\| / 3$ on $\left\{z \in \mathbf{C}: c_{n} / 2<|z|<2 c_{n}\right\}$. Now with $M$ fixed, the sum contains at 
most $(2 \log M) / \log (1 / c)$ terms, each of which has variation less than $2\|f\| / R$ on $\mathrm{C} \backslash \cup S\left(c_{m}, R r_{n}\right)$. If $R$ is chosen such that $(4 \log M) / R \log (1 / c)<\varepsilon / 3$ and $\delta>0$ is chosen to guarantee that the disks $S\left(c_{n}, R r_{n}\right)$ are mutually disjoint for $\left|c_{n}\right|<\delta$, then $R$ and $\delta$ work in (3) and (4).

Combining Theorem 3.4 with the results of $\S 2$, we can characterize the interpolating sequences for the class of $L$-domains we are considering.

THEOREM 3.6. Let $\mathscr{D}=\Delta \backslash\{0\} \backslash \cup \Delta_{n}$ with $\sup \left(c_{n+1} / c_{n}\right)<1$ and $\Sigma\left(r_{n} / c_{n}\right)^{p}<\infty$, some $p \geqslant 1$. Let $R>1$ be such that the sets $\Delta_{0}=\{z \in \mathrm{C}: 1 / R \leqslant|z|<1\}$ and $\overline{S\left(c_{n}, R r_{n}\right)}, n=1,2, \ldots$, are mutually disjoint. Let $T_{n}(z)=r_{n} /\left(z-c_{n}\right)$. Then $a$ subset $S \subset \mathscr{D}$ interpolates $H^{\infty}(\mathscr{D})$ if and only if it is $\pi$-separated and

$$
\delta\left(S \cap \Delta_{0}\right) \wedge \inf _{n} \delta\left(T_{n}\left(S \cap S\left(c_{n}, R r_{n}\right)\right)\right)>0 .
$$

Proof. An $H^{\infty}(\mathscr{D})$ interpolating sequence is certainly $\pi$-separated. This and Lemma 3.3. prove sufficiency in Theorem 3.6.

Let $S \subset \mathscr{D}$ be a set with

$$
\inf \{\pi(z, w): z, w \in S, z \neq w\} \geqslant \varepsilon^{\prime}>0
$$

and

$$
\delta\left(S \cap \Delta_{0}\right) \wedge \inf _{n} \delta\left(T_{n}\left(S \cap S\left(c_{n}, R r_{n}\right)\right)\right)>0 .
$$

Choose $R$ and $\delta$ by Lemma 3.5, using the value $\varepsilon^{\prime} / M$ for $\varepsilon$. Let $S_{1}=S \cap$ $\left(\cup_{c_{n} \leqslant \delta} S\left(c_{n}, R r_{n}\right) \cup\{z:|z| \geqslant \delta\}\right)$ and $S_{2}=S \backslash S_{1}$. By Theorem 3.4, $S_{1}$ is an $H^{\infty}(\mathscr{D})$ interpolating sequence. Let $S_{3}=\left\{-|z|: z \in S_{2}\right\}$. If $z, w \in S_{2}$, then

$$
\pi(-|z|,-|w|) \geqslant \pi(z, w)-[\pi(-|z|, z)+\pi(-|w|, w)] \geqslant \varepsilon^{\prime}-2 \varepsilon^{\prime} / M>\varepsilon^{\prime} / 2,
$$

where $z \neq w$ and $M$ is chosen greater than 4. Let $f(z)=\int_{E} d x /(x-z)$. By Corollary 2.6, $f\left(S_{3}\right)$ is interpolating for $H^{\infty}(\mathscr{E}), \mathscr{E}$ the horizontal strip of $\S 2$. Let

$$
\eta=\inf \left\{\pi(f(z), f(w)): z, w \in S_{3}, z \neq w\right\} .
$$

Now $\pi(f(-|z|), f(z)) \leqslant \pi(-|z|, z) \leqslant \varepsilon^{\prime} / M$. If $\varepsilon^{\prime} / M<\eta / 3$, then $f\left(S_{2}\right)$ has exactly one point in the disk of $\pi$-radius $\varepsilon^{\prime} / M$ about each point of $f\left(S_{3}\right)$. If $\varepsilon^{\prime} / M$ is sufficiently small, i.e. $M$ is large, it follows from Lemma 4.2 of [7] that $f\left(S_{2}\right)$ interpolates for $H^{\infty}(\mathscr{E})$ and, hence, $S_{2}$ interpolates for $H^{\infty}(\mathscr{D})$.

Let $h(z)=\sum\left(r_{n} /\left(z-c_{n}\right)\right)^{p}-\sum\left(r_{n} / c_{n}\right)^{p} \in H^{\infty}(\mathscr{D})$. Then

$$
\lim _{\substack{z \in 0 \\ z \in S_{1}}}|h(z)| \geqslant\left(\frac{1}{R}\right)^{p} \text { and } \lim _{\substack{z \rightarrow 0 \\ z \in S_{2}}}|h(z)| \leqslant\left(\frac{1}{R}\right)^{p} \text {. }
$$

We have shown that $S_{1}$ and $S_{2}$ separately interpolate for $H^{\infty}(\mathscr{D})$. Hence, $S_{1}^{-\mathscr{M}(\mathscr{D})}$

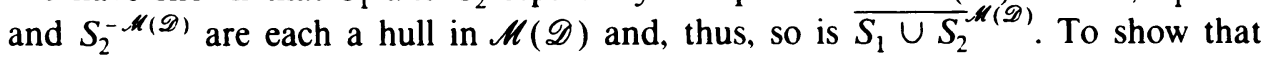
$S=S_{1} \cup S_{2}$ interpolates for $H^{\infty}(\mathscr{D})$, it now suffices, by the Badé-Curtis Theorem, to show that if $T \subset S$, then $T^{-\mu(D)} \cap(S \backslash T)^{-\mu(D)}=\varnothing$.

Suppose not. Let $m \in T^{-\mathscr{M}(\mathscr{D})} \cap(S \backslash T)^{-\mathscr{M}(\mathscr{D})}$. Clearly $\hat{z}(m)=0$. If $|\hat{h}(m)|>$ $(1 / R)^{p}$, then $m \in\left(S_{1} \cap T\right)^{-\mathscr{M}(\mathscr{D})} \cap\left(S_{1} \backslash T\right)^{-\mathscr{M}(\mathscr{D})}$, and if $|\hat{h}(m)|<(1 / R)^{p}$, then $m \in\left(S_{2} \cap T\right)^{-\mathscr{M}(\mathscr{D})} \cap\left(S_{2} \backslash T\right)^{-\mathscr{M}(\mathscr{D})}$, both of which are impossible. Suppose $|\hat{h}(m)|$ $=(1 / R)^{p}$. Let $S_{4}=\left\{z \in S: \frac{1}{2} R r_{n}<\left|z-c_{n}\right|<2 R r\right.$, some $\left.n \geqslant 1\right\}$. Then $S_{4}$ is interpolating, but $m \in\left(S_{4} \cap T\right)^{-\mathscr{M}(\mathscr{D})} \cap\left(S_{4} \backslash T\right)^{-\mathscr{M}(\mathscr{D})}$, a contradiction. 
S. Fisher has pointed out that if 0 is not a regular point for the Dirichlet problem on $\mathscr{D}$, then a sequence $\left\{\alpha_{n}\right\}$ converging to 0 on the negative axis can be constructed which is an interpolating sequence for the bounded harmonic functions on $\mathscr{D}$, but is not interpolating for $H^{\infty}(\mathscr{D})$ (see [5, p. 191]).

THEOREM 3.7. Let $\mathscr{D}=(\Delta \backslash\{0\}) \backslash \cup \Delta_{n}$ with $\sup \left(c_{n+1} / c_{n}\right)<1$ and $\Sigma\left(r_{n} / c_{n}\right)^{p}<$ $\infty$, some $p \geqslant 1$. The sequences in $\mathscr{D}$ which interpolate for bounded harmonic functions on $\mathscr{D}$ are precisely the sequences in $\mathscr{D}$ which interpolate for $H^{\infty}(\mathscr{D})$ if and only if $\sum\left(\log c_{n} / \log r_{n}\right)=\infty$.

Proof. By Theorem III.62 of [8], 0 is a regular point for the Dirichlet problem on $\mathscr{D}$ if and only if $\sum n / \log \left(1 / \gamma_{n}\right)=\infty$, where $\gamma_{n}$ is the logarithmic capacity of $\{z \in \mathbf{C}$ : $\left.\lambda^{n+1} \leqslant|z| \leqslant \lambda^{n}\right\} \backslash \mathscr{D}$. For an appropriate choice of $\lambda$, and $n$ large, this annulus will contain at most one $c_{k}$. Then $\gamma_{n}=\gamma\left(S\left(c_{k}, r_{k}\right)\right)=r_{k}$ and $\lambda^{n+1} \leqslant c_{k} \leqslant \lambda^{n}$, so

$$
n \log (1 / \lambda) \leqslant \log c_{k} \leqslant(n+1) \log (1 / \lambda) .
$$

Thus $\sum n / \log \left(1 / \gamma_{n}\right)=\infty$ if and only if $\sum\left(\log c_{n} / \log r_{n}\right)=\infty$. The observation of Fisher now gives the necessity in Theorem 3.7. If 0 is a regular point for the Dirichlet problem then every bounded harmonic function has a limit as $r$ goes to 0 along the negative axis. It follows that if $S$ interpolates bounded harmonic functions in $\mathscr{D}$, then $S \cap S(0, \delta) \subset \bigcup\left\{S\left(c_{n}, R r_{n}\right):\left|c_{n}\right| \leqslant \delta\right\}$ for some $\delta>0$, since it is easily seen that any bounded harmonic function has small variation on $S(0, \delta) \backslash \cup S\left(c_{n}, R r_{n}\right)$. But now each $T_{n}\left(S\left(c_{n}, R r_{n}\right) \cap S\right), T_{n}(z)=r_{n} /\left(z-c_{n}\right)$, interpolates values in $\{0,1\}$ by a function harmonic on $\left\{z \in \mathrm{C}: 1 / R_{n}<|z|<1\right\}$ and bounded by a constant $M$. For large $R_{n}$, the harmonic extension to $\Delta$ of the boundary values of these functions on $\partial \Delta$ interpolate values which are less than $1 / 10$ on a given subset of $T_{n}\left(S\left(c_{n}, R r_{n}\right) \cap S\right)$ and greater than $9 / 10$ on the complement of this subset. We want to show that inf $\delta\left(T_{n}\left(S\left(c_{n}, R r_{n}\right) \cap S\right)\right)>0$. Garnett showed in [5] that $\delta\left(T_{n}\left(S\left(c_{n}, R r_{n}\right) \cap S\right)\right)>0$ if and only if there is a constant $A_{n}$ such that $\mu(X) \leqslant A_{n} \cdot \eta$ where $X=\left\{r e^{i \theta} \in \Delta: r \geqslant 1-\eta,\left|\theta-\theta_{0}\right|<\eta\right\}$ and $\mu$ is the measure with mass $1-|z|^{2}$ at each point $z \in T_{n}\left(S\left(c_{n}, R r_{n}\right) \cap S\right)$. It suffices to show that inf $A_{n}>0$. In the proof of the theorem in [5], Garnett actually proves that the constant $A$ depends only on the $M$ in his Lemma 1 . He uses the functions of his Lemma 1, Part 2, only in the proof of his Lemma 3 and he does not use the full properties of these functions. In fact, harmonic functions taking values less than $1 / 10$ on a given subset of the sequence and values greater than $9 / 10$ on the complement of this subset, and bounded by $M$, will do just fine. This means that $A_{n}$ depend only on $M$ and we are done.

The proof of Theorem 3.7 also shows that the following result is true.

THEOREM 3.8. Let $\mathscr{D}=(\Delta \backslash\{0\}) \backslash \cup \Delta_{n}$ with $\sup \left(c_{n+1} / c_{n}\right)<1$ and $\Sigma\left(r_{n} / c_{n}\right)^{p}<$ $\infty$, some $p \geqslant 1$. A sequence $S \subset \mathscr{D}$ interpolates for $H^{\infty}(\mathscr{D})$ if and only if it interpolates for the bounded harmonic functions on $\mathscr{D}$ and is $\pi$-separated.

4. Tangential homomorphisms. Let $\mathscr{D}=\mathscr{E} \backslash \cup \Delta_{n}$ be such that there are mutually disjoint disks $D_{n}$ contained in $\mathscr{E}$ with center $D_{n}=c_{n}=$ center $\Delta_{n}$ and $\sum\left(\operatorname{rad} \Delta_{n} / \operatorname{rad} D_{n}\right)^{p}<\infty$ for some $p \geqslant 1$. 
Let $\mathscr{T}=\bigcap_{N, R} \overline{U_{N}^{x} S\left(c_{n}, R r_{n}\right)^{\mu}(\mathscr{D})}$ where $R>1$ and $r_{n}=\operatorname{rad} \Delta_{n}$. The homomorphisms in $\mathscr{T}$ are in the fiber at 0 and they are "tangential", that is, are approached by nets in $\mathscr{D}$ which lie close to $\cup \Delta_{n}$. We will show that each nontrivial Gleason part in $\mathscr{T}$ is an analytic disk and that a point in $\mathscr{T}$ lies in a nontrivial Gleason part if and only if it is contained in the closure of an interpolating sequence. To do this we will show that the factorization theorems used by Hoffman in [7] generalize to this setting.

We begin with the following simple technical lemma which can be used to construct analytic functions on $\mathscr{D}=\mathscr{E} \backslash \bigcup \Delta_{n}$. Let $T_{n}(z)=r_{n} /\left(z-c_{n}\right)$.

Lemma 4.1. Let $M, \varepsilon>0$ be given. Then there is an $N$ such that the following properties hold. If $f_{n} \in H^{\infty}(\Delta)$ with $\left\|f_{n}\right\| \leqslant M, n=1,2, \ldots$, then:

(a) $\sum_{N}^{\infty}\left(z^{p} f_{n}\right) \circ T_{n} \in H^{\infty}(\mathscr{D})$;

(b) $\left\|\sum_{N}^{\infty}\left(z^{p} f_{n}\right) \circ T_{n}\right\| \leqslant M+\varepsilon$;

(c) $\left|\sum_{N}^{\infty}\left(z^{p} f_{n}\right) \circ T_{n}\right|<\varepsilon$ on $\mathbf{C} \backslash \bigcup_{N}^{\infty} D_{n}$; and

(d) $\left|\sum_{N}^{\infty}\left(z^{p} \circ f_{n}\right) \circ T_{n}-z^{p} f_{k} \circ T_{k}\right|<\varepsilon$ on $D_{k}, k \geqslant N$.

Proof. The lemma is an immediate consequence of the inequality $\left|z^{p} f_{n} \circ T_{n}\right| \leqslant$ $M\left(\operatorname{rad} \Delta_{n} / \operatorname{rad} D_{n}\right)^{p}$ on $\mathrm{C} \backslash D_{n}$.

The next lemma, which is also elementary, says that the metric $\pi$ is easily described locally near $\mathscr{T}$.

Lemma 4.2. Let $T_{n}^{-1}\left(\psi_{0}\right)$ be the metric on $S\left(c_{n}, R r_{n}\right)$ obtained by lifting $\psi_{0}$ along $T_{n}^{-1}$. Given $R$ and $r<1$ there is an $N$ such that for $n \geqslant N, r \pi \leqslant T_{n}^{-1}\left(\psi_{0}\right) \leqslant \pi$ on $S\left(c_{n}, R r_{n}\right)$.

Proof. Since $T_{n} \in H^{\infty}(\mathscr{D})$, it must be that $T_{n}^{-1}\left(\psi_{0}\right) \leqslant \pi$. For $1 / R<|z|<1$ and $r$ so large that $R \leqslant R_{n}$,

$$
f \circ T_{n}^{-1}=\frac{1}{2 \pi i} \int_{\partial \Delta} \frac{f \circ T_{n}^{-1}(\xi) d \xi}{\xi-z}-\frac{1}{2 \pi i} \int_{|=|=1 / R_{n}} \frac{f \circ T_{n}^{-1}(\xi) d \xi}{\xi-z},
$$

where $f \in H^{\infty}(\mathscr{D})$ and $\|f\| \leqslant 1$. There is a constant $k=k(R)$ such that $|z-w| \leqslant$ $k \pi(z, w)$ for $1 / R \leqslant|z|,|w|<1$. Schwarz's lemma on $\left\{z \in \mathbf{C}:|z|>1 / R_{n}\right\}$ shows that the second integral in the above expression differs by less than $\left(k / R_{n}\right) \pi(z, w)$ when evaluated at $z$ and $w$. Hence the Carathéodory metric of $T_{n}(\mathscr{D})$ is less than $\left(1+k / R_{n}\right) \psi_{0}$ on $\{z \in \mathbf{C}: 1 / R<|z|<1\}$.

Fix $R>1$ such that the $S\left(c_{n}, R r_{n}\right)$ are mutually disjoint and let $S$ be a sequence in US $\left(c_{n}, R r_{n}\right)$. Write $\delta(S)=\inf \delta\left(T_{n}\left(S \cap S\left(c_{n}, R r_{n}\right)\right)\right)$, so that $\delta(S)>0$ if and only if $S$ is an interpolating sequence for $H^{\infty}(\mathscr{D})$. By Theorem 3.2 of Hoffman [7], each sequence $S$ in $\Delta$ with $\delta(S)>0$ can be factored, $S=S_{1} \cup S_{2}$, such that $\delta\left(S_{i}\right) \geqslant \delta(S)^{1 / 2}, i=1,2$. Factoring each $T_{n}\left(S \cap S\left(c_{n}, R r_{n}\right)\right)$ gives the following result.

Lemma 4.3. Let $S \subset \cup S\left(c_{n}, R r_{n}\right)$ with $\delta(S)>0$. Then there are disjoint $S_{1}, S_{2}$ such that $S=S_{1} \cup S_{2}$ and $\delta\left(S_{i}\right) \geqslant \delta(S)^{1 / 2}, i=1,2$.

Let $S$ be a sequence in $U S\left(c_{n}, R r_{n}\right)$. If $T_{n}\left(S \cap \Delta\left(c_{n}, R r_{n}\right)\right)$ is a Blaschke sequence, we may form the Blaschke product $A_{n}$ with zeros at the points of $T_{n}\left(S \cap \Delta\left(c_{n}, R r_{n}\right)\right)$. 
By Lemma 1, the function $\Omega_{N}=\sum_{N}^{\infty}\left(z^{p} A_{n}\right) \circ T_{n}$ is in $H^{\infty}(\mathscr{D})$ and satisfies $\left\|\Omega_{N}\right\| \leqslant 1$ $+\varepsilon$ and $\left|\Omega_{N}(z)\right|<\varepsilon$ for $z \in S$, if $N$ is sufficiently large.

The following lemma states a well-known property of interpolating Blaschke products. It is essentially Lemma 4.2 of Hoffman [7].

Lemma 4.4. Let $M, \varepsilon$ be given. Then there is $a \delta<1$ such that if $A$ is a Blaschke product with $\delta(A) \geqslant \delta$ and $\alpha \in \Delta$ with $A(\alpha)=0$, then there is a $c$ with $|c|=1$ such that

$$
\left|A(z)-c \frac{\alpha-z}{1-\bar{\alpha} z}\right|<\varepsilon \text { on } S_{\psi_{0}}(\alpha, M)
$$

and

$$
|A| \geqslant 1-\varepsilon \text { on } \Delta \backslash S_{\psi_{0}}\left(A^{-1}(0), M\right),
$$

where $S_{\psi_{0}}(z, R)=\left\{w: \psi_{0}(w, z)<R\right\}$ and $\psi_{0}$ is the Poincare metric on the open unit $\operatorname{disc} A$.

Combining Lemmas 4.1, 4.2 and 4.4, we obtain a lemma for the functions $\Omega_{N}$ which is similar to Lemma 4.4.

LemMA 4.5. Let $\varepsilon>0, M$ be given. There are $R, N$, and $\delta$ such that if $S \subset$ $\cup_{N}^{\infty} S\left(c_{n}, R r_{n}\right)$ and $w \in S$, then there is a c with $|c|=1$,

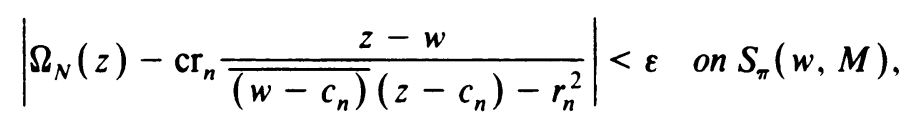

and

$$
\left|\Omega_{N}\right| \geqslant 1-\varepsilon \text { on } \bigcup_{N}^{\infty} S\left(c_{n}, R r_{n}\right) \backslash S_{\pi}(S, M),
$$

where $S_{\pi}(w, M)=\{z: \pi(z, w)<M\}$ and $S_{\pi}(S, M)=\bigcup\{S \pi(w, M): w \in S\}$.

Proof. Let $L_{\alpha}(z)=(\alpha-z)(1-\bar{\alpha} z)$. The Blaschke product $A_{n}$ vanishes at $T_{n}(w)$, so by Lemma 4.4 there is a $c$ with $|c|=1,\left|A_{n}\left(T_{n}(z)\right)-c L_{T_{n}\left({ }^{\prime}\right)}\left(T_{n}(z)\right)\right|<\varepsilon / 10$ on $S_{\pi}(w, M)$, and $\left|A_{n}\left(T_{n}(z)\right)\right|>1-\varepsilon / 10$ on $S\left(c_{n}, R r_{n}\right) \backslash S_{\pi}(S, M)$, where $n, \delta$ are sufficiently large. Choosing $R$ close to 1 forces $z^{p}$ to be close to a constant of modulus 1 on $S_{\pi}(w, M)$. Using Lemma 4.1(d), and noticing that

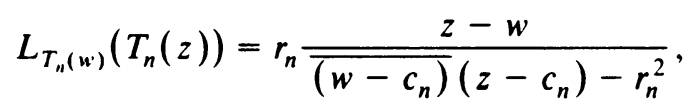

we are done.

Hoffman shows that analytic disks occur in $\mathscr{M}(\Delta)$ using the following scheme: If $S$ is an interpolating sequence in $\Delta, m \in S^{-\mathcal{M}(\Delta)}$, and $\left\{z_{\lambda}\right\}$ is a net in $S$ which converges to $m$, let $L_{\lambda}(z)=\left(z_{\lambda}-z\right) /\left(\perp-\bar{z}_{\lambda} z\right)$. Consider $\left\{L_{\lambda}\right\}$ to be a net in $\mathscr{M}(\Delta)^{\Delta}$ equipped with the compact open topology. Then $\left\{L_{\lambda}\right\}$ converges to an injective analytic mapping of $\Delta$ into $\mathscr{M}(\Delta)$ which covers $m$.

A similar scheme works for $\mathscr{T}$. 
THEOREM 4.6. Let $m \in \mathscr{T}, S \subset \bigcup_{N}^{\infty} S\left(c_{n}, R r_{n}\right)$ an interpolating sequence, and $\left\{z_{\lambda}\right\} a$ net in $S$ with $\lim z_{\lambda}=m$. For $z_{\lambda} \in S\left(c_{n}, R r_{n}\right)$, let

$$
F_{\lambda}(z)=\frac{\left[c_{n} \overline{\left(z_{\lambda}-c_{n}\right)}+r_{n}^{2}\right] z-r_{n} z_{\lambda}}{\left[\overline{\left(z_{\lambda}-c_{n}\right)}-r_{n}\right] z-r_{n}} \text {. }
$$

Then

(1) each compact subset of $\Delta$ is eventually contained in $F_{\lambda}^{-1}(\mathscr{D})$;

(2) for each $f \in H^{\infty}(\mathscr{D}), f \circ F_{\lambda}$ converges uniformly on compact subsets of $\Delta$ to an analytic function defined on $\Delta$; and

(3) the mapping $F_{m}$ of $\Delta$ into $\mathscr{M}(\mathscr{D})$, defined by $F_{m}(z)(f)=\lim _{\lambda} f \circ F_{\lambda}(z)$, is an injective analytic mapping into $\mathscr{M}(\mathscr{D})$ satisfying $F_{m}(0)=m$.

Proof. (1) The function $F_{\lambda}$ maps $\Delta$ conformally on $\mathscr{S} \backslash S\left(c_{n}, r_{n}\right)$, where $\mathscr{S}$ is the Riemann sphere, and takes 0 into $z_{\lambda}$. Since $F_{\lambda}^{-1}(\infty) \rightarrow-1,(1)$ is immediate.

(2) Using Pick's theorem and compactness, it suffices to prove that $f \circ F_{\lambda}$ converges at each point of $\Delta$. For $z_{0} \in \Delta, c \in \mathbf{C}$, and $\varepsilon>0$, let $S^{\prime}=\left\{z_{\lambda} \in S\right.$ : $F_{\lambda}\left(z_{0}\right) \in \mathscr{D}$ and $\left.\left|f\left(F_{\lambda}\left(z_{0}\right)\right)-c\right|<\varepsilon\right\}$. Since $S$ is interpolating, either $z_{\lambda}$ is eventually in $S^{\prime}$ or $z_{\lambda}$ is eventually in $S \backslash S^{\prime}$, i.e. either eventually $\left|f \circ F_{\lambda}\left(z_{0}\right)-c\right|<\varepsilon$ or eventually $\left|f \circ F_{\lambda}\left(z_{0}\right)-c\right| \geqslant \varepsilon$.

(3) That $F_{m}$ is analytic means $\hat{f} \circ F_{m}=\lim _{\lambda} f \circ F_{\lambda}$ is analytic for each $f \in H^{\infty}(\mathscr{D})$. Also

$$
F_{m}(0)(f)=\lim _{\lambda} f \circ F_{\lambda}(0)=\lim _{\lambda} f\left(z_{\lambda}\right)=m(f) .
$$

It remains to show that $F_{m}$ is injective. By Lemma 4.5(1), if $r<1$ and $\varepsilon>0$ are given, there is an $N$ such that $\left|\Omega_{N} \circ F_{\lambda}(z)-c_{\lambda} z\right|<\varepsilon$ for $|z|<r$ and some $c_{\lambda} \in \mathbf{C}$ with $\left|c_{\lambda}\right|=1$. Choosing a subnet such that $\lim c_{\lambda}=c$, then $\left|\hat{\Omega}_{N} \circ F_{m}(z)-c z\right|<\varepsilon$ for $|z|<r$, where $|c|=1$. Since $\varepsilon>0$ is arbitrary, we are done.

THEOREM 4.7. Let $m \in \mathscr{T}$ be contained in the closure of an interpolating sequence in $\mathscr{D}$. Then $\mathscr{G}(m)$ is an analytic disk.

Proof. By Theorem 4.6, $F_{m}(\Delta)$ is an analytic disk containing $m$. That $\mathscr{T} \cap \mathscr{G}(m)$ $=F_{m}(\Delta)$ follows immediately from Lemma $4.5(2)$ in exactly the same way that the corresponding result in the disk follows from Lemma 4.2 of [7]. We will prove a corona theorem (Theorem 4.11) later which will guarantee that $\mathscr{G}(m) \subset \mathscr{T}$.

Let $\lambda \in \partial \mathscr{D}$. For $A_{n}$ a sequence of Blaschke products, define

$$
\Omega_{N, \lambda}=\sum_{N}^{\infty}\left(z^{p} A_{n}\right) \circ T_{n}-\sum_{N}^{\infty}\left(z^{p} A_{n}\right) \circ T_{n}(\lambda) .
$$

The following lemma states some elementary properties of $\Omega_{N, \lambda}$ on $\mathscr{M}_{\lambda}(\mathscr{D}) \cap \mathscr{T}$.

LEMMA 4.8. (1) $\Omega_{M, \lambda}=\Omega_{N, \lambda}$ on $\mathscr{T} \cap \mathscr{M}_{\lambda}(\mathscr{D})$, all $M, N$.

(2) If each $A_{n}=A_{n}^{(1)} A_{n}^{(2)}$ and $\Omega_{N . \lambda}^{(i)}$ corresponds to $\left\{A_{n}^{(i)}\right\}$, then $\left|\Omega_{N . \lambda}\right|=\left|\Omega_{N . \lambda}^{(1)}\right|\left|\Omega_{N . \lambda}^{(2)}\right|$ on $\mathscr{T} \cap \mathscr{M}_{\lambda}(\mathscr{D})$.

(3) If $S \subset \bigcup_{N}^{x} S\left(c_{n}, R r_{n}\right)$ is interpolating and $\Omega_{N, \lambda}$ is the corresponding function, then for $m \in \mathscr{T} \cap \mathscr{M}_{\lambda}(\mathscr{D}), \hat{\Omega}_{N, \lambda}(m)=0$ if and only if $m \in S^{-\mathscr{M}(\mathscr{D})}$. 
Proof. (1) and (2) are elementary consequences of Lemma 4.1. From Lemma 4.5 it is evident that if $S$ is interpolating, then $\Omega_{N, \lambda}$ is bounded away from 0 on $\cup_{N}^{\infty} S\left(c_{n}, R r_{n}\right) \backslash S_{\pi}(S, M)$, where $M>0$ is given and then $N$ is chosen sufficiently large. Hence if $m \in \mathscr{T} \cap \mathscr{M}_{\lambda}(\mathscr{D})$ and $m$ is contained in the closure

$$
\bigcup_{N}^{\infty} S\left(c_{n}, R r_{n}\right) \backslash S_{\pi}(S, M),
$$

then $\hat{\Omega}_{N, \pi}(m) \neq 0$. Thus $\hat{\Omega}_{N, \pi}(m)=0$ implies that $m$ is in the closure of $S_{\pi}(S, M)$ for all $M>0$. If $M$ is chosen so small that $\pi(z, w)<M$ implies $|f(z)-f(w)|<\varepsilon / 2$ for all $f \in H^{\infty}(\mathscr{D}),\|f\| \leqslant 1$, then the neighborhood of $m$ defined by $\left\{n: \mid \hat{f}_{i}(n)-\right.$ $\left.\hat{f}_{i}(m) \mid<\varepsilon / 2,\left\|f_{i}\right\| \leqslant 1, i=1, \ldots, k\right\}$ contains a point of $S_{\pi}(S, M)$ implies that the neighborhood of $m$ defined by $\left\{n:\left|\hat{f}_{i}(n)-\hat{f}_{i}(m)\right|<\varepsilon, i=1, \ldots, k\right\}$ contains a point of $S$. Thus $\hat{\Omega}_{N, \lambda}(m)=0$ implies that $m$ is contained in the closure of $S$ in $\mathscr{M}(\mathscr{D})$. The opposite implication follows from Lemma 4.1 .

With these properties of the $\Omega_{N . \lambda}$ in hand, we can now generalize a crucial theorem of Hoffman [7, Theorem 5.2] to our context.

THEOREM 4.9. Let $S \subset \bigcup_{N}^{\infty} S\left(c_{n}, R r_{n}\right)$ be such that each $T_{n}\left(S \cap S\left(c_{n}, R r_{n}\right)\right)$ is a Blaschke sequence, and let $\Omega_{N, \lambda}$ be the corresponding function. Let $m \in \mathscr{T} \cap \mathscr{M}_{\lambda}(\mathscr{D})$ with $\hat{\Omega}_{N, \lambda}(m)=0$. Then either $m$ is in the closure of an interpolating subsequence of $S$, or $S$ can be factored, $S=S_{1} \cup S_{2}, S_{1} \cap S_{2}=\varnothing$, such that the corresponding functions $\Omega_{N, \lambda}^{(1)}$ and $\Omega_{N, \lambda}^{(2)}$ satisfy $\widehat{\Omega_{N, \lambda}^{(1)}(m)}=\widehat{\Omega_{N, \gamma}^{(2)}(m)}=0$.

Proof. Let $M \geqslant 0$. Apply Theorem 5.2 of [7] to the Blaschke product $A_{n}$ of $T_{n}\left(S \cap S\left(c_{n}, R r_{n}\right)\right)$ to get $A_{n}=A_{n}^{(1)} A_{n}^{(2)}$, which satisfy $a\left|A_{n}^{(1)}\right|^{1 / b} \leqslant\left|A_{n}^{(2)}\right| \leqslant a^{-1}\left|A_{n}^{(1)}\right|^{b}$, for positive constants $a$ and $b$ not depending on $n$, on

$$
\Delta \backslash S_{\psi_{0}}\left(T_{n}\left(S \cap S\left(c_{n}, R r_{n}\right)\right), M\right) \text {. }
$$

Form the corresponding functions $\Omega_{N, \lambda}^{(1)}$ and $\Omega_{N, \lambda}^{(2)}$. Clearly if $m \in \mathscr{T} \cap \mathscr{M}_{\lambda}(\mathscr{D})$ is in the closure of $\bigcup_{N}^{\infty} S\left(c_{n}, R r_{n}\right) \backslash S_{\pi}(S, M)$, then

$$
a\left|\widehat{\Omega_{N, \lambda}^{(1)}(m)}\right|^{1 / h} \leqslant\left|\widehat{A_{N, \lambda}^{(2)}(m)}\right| \leqslant \frac{1}{a}\left|\widehat{\Omega_{N . \lambda}^{(1)}(m)}\right|^{h} .
$$

Hence if $\widehat{\Omega_{N, \lambda}(m)}=0$, considering Lemma $4.8(2)$, we have $\widehat{\Omega_{N . \lambda}^{(1)}(m)}=$ $\widehat{\Omega_{N . \lambda}^{(2)}(m)}=0$.

Suppose $m \in \mathscr{T} \cap \mathscr{M}_{\lambda}(\mathscr{D})$ is in the closure of $S_{\pi}(S, M)$ for every $M>0$. Factor the Blaschke product $A_{n}$ of $T_{n}\left(S \cap S\left(c_{n}, R r_{n}\right)\right)$ into $A_{n}=A_{n}^{(1)} A_{n}^{(2)}$ by Theorem 3.2 of [7] and form the functions $\Omega_{N, \lambda}^{(1)}$ and $\Omega_{N, \lambda}^{(2)}$. If $\widehat{\Omega_{N, \lambda}^{(1)}(m)} \neq \varnothing$, let $T=\{z \in S$ : $\left.\left|\Omega_{N . \lambda}^{(1)}(z)\right| \geqslant \frac{1}{2}\left|\widehat{\Omega_{N . \lambda}^{(1)}(m)}\right|\right\}$. For $M$ sufficiently small, $\left|\Omega_{N . \lambda}^{(1)}\right| \leqslant \frac{3}{4}\left|\widehat{\Omega_{N . \lambda}^{(1)}(m)}\right|$ on

$$
S_{\pi}(S \backslash T, M),
$$

so $m$ is in the closure of $S_{\pi}(T, M)$ for all sufficiently small $M$. Let $A_{n}^{(3)}$ be the Blaschke product of $T_{n}\left(T \cap S\left(c_{n}, R r_{n}\right)\right)$. We may assume $\left|A_{n}^{(1)}(\alpha)\right| \geqslant \frac{1}{4}\left|\Omega_{N . \lambda}^{(1)}(m)\right|$ for $\alpha \in T_{n}\left(T \cap S\left(c_{n}, R r_{n}\right)\right)$, and hence also $A_{n}^{(2)}(\alpha)=0$ for these $\alpha$ 's. By Theorem 3.2 of [7],

$$
\left(1-|\alpha|^{2}\right)\left|A_{n}^{(3) \prime}(\alpha)\right| \geqslant\left(1-|\alpha|^{2}\right)\left|A_{n}^{(2)}(\alpha)\right| \geqslant\left|A_{n}^{(1)}(\alpha)\right| \geqslant \frac{1}{4}\left|\widehat{\Omega_{N . \lambda}^{(1)}(m)}\right| .
$$


It follows that $T$ is an interpolating sequence. Since $m$ is in the closure of $S_{\pi}(T, M)$ for all sufficiently small $M$, it must be that $\overline{\Omega_{N . \lambda}^{(3)}(m)}=0$, and hence, by Lemma 4.8(3), $m$ is in the closure $T$.

Lemma 4.10. Let $f \in H^{\infty}(\mathscr{D})$ with $\|f\| \leqslant 1$. Then there is a function $g \in H^{\infty}(\mathscr{D})$ with $\|g\| \leqslant 1+\varepsilon$ and a function $\Omega_{N, \lambda} \in H^{\infty}(\mathscr{D})$ such that $|\hat{f}|=\left|\left(\overline{g^{2} \Omega_{N . \lambda}}\right)\right|$ on $\mathscr{T} \cap$ $\mathscr{M}_{\lambda}(\mathscr{D})$.

Proof. Let

$$
h_{n}=\frac{1}{2 \pi i} \int_{\partial \Delta} \frac{f \circ T_{n}^{-1}(\xi) d \xi}{\xi-z} .
$$

Let $h_{n}=g_{n}^{2} A_{n}$, where $g_{n}$ is never zero and $A_{n}$ is a Blaschke product. Let

$$
g=\sum_{N}^{x}\left(z^{p} g_{n}\right) \circ T_{n}-\sum_{N}^{x} z^{p} g_{n} \circ T_{n}(\lambda)
$$

and

$$
\Omega_{N . \lambda}=\sum_{N}^{\infty}\left(z^{p} A_{n}\right) \circ T_{n}-\sum_{N}^{\infty}\left(z^{p} A_{n}\right) \circ T_{n}(\lambda) .
$$

Just as in Lemma 4.8(2), elementary calculations with Lemma 4.1 show that $|\hat{f}|=$ $\left|\left(\overline{g^{2} \Omega_{N . \lambda}}\right)\right|$ on $\mathscr{T} \cap \mathscr{M}_{\lambda}(\mathscr{D})$.

For $\lambda \in \partial \mathscr{D}$, let $I_{\lambda}=\left\{f \in H^{\infty}(\mathscr{D})\right.$ : given $\varepsilon>0$ there are $R$ and $\delta>0$ such that $|f(z)|<\varepsilon$ on $S\left(\lambda, \delta^{\prime}\right) \backslash \bigcup_{\left|c_{n}\right|<\delta^{\prime}} S\left(c_{n}, R r_{n}\right)$ for all $\left.\delta^{\prime} \leqslant \delta\right\}$. It is easy to see that $I_{\lambda}$ is equal to the seemingly less restrictive set $\left\{f \in H^{\infty}(\mathscr{D}): \lim _{z \rightarrow \lambda} f(z)=0\right.$, where $z$ goes to $\lambda$ through the set $\left.\mathscr{E} \backslash \cup D_{n}\right\}$. Using the ideal $I_{\lambda}$, we can state a little corona theorem for $\mathscr{D}=\mathscr{E} \backslash \cup \Delta_{n}$.

THEOREM 4.11. Let $m \in \mathscr{M}_{\lambda}(\mathscr{D})$. If $m$ is not in the closure of $\mathscr{D}$ in $\mathscr{M}(\mathscr{D})$, then $I_{\lambda}$ is contained in the kernel of $m, I_{\lambda} \subset \operatorname{Ker}(m)$.

Proof. Let $h \in I_{\lambda}$. Suppose $m \in H^{\infty}(\mathscr{D})$ is not in the closure of $\mathscr{D}$. Then there are $f_{1}, \ldots, f_{n} \in H^{\infty}(\mathscr{D})$ and $\varepsilon>0$ such that $\sum_{1}^{n}\left|f_{i}\right| \geqslant \varepsilon$ on $\mathscr{D}$ and $m\left(f_{i}\right)=0, i=$ $1, \ldots, n$. Let $T_{k}(z)=r_{k} /\left(z-c_{k}\right)$, let

$$
f_{i, k}(z)=\frac{1}{2 \pi i} \int_{\partial \Delta} \frac{f_{i} \circ T_{k}^{-1}(\xi) d \xi}{\xi-z},
$$

and let

$$
h_{k}(z)=\frac{1}{2 \pi i} \int_{\partial د} \frac{h^{p} \circ T_{k}^{-1}(\xi) d \xi}{\xi-z} .
$$

Elementary estimates show that (1) $f_{i, k}, h_{k} \in H^{\infty}(\Delta)$ are uniformly bounded, (2) for large $k, \sum_{i}\left|f_{i, k}\right| \geqslant \varepsilon / 2$ on $\Delta$, and (3) for large $k,\left|h_{k}(z)\right| \leqslant 2\|h\|\left(r_{k} / R_{k}\right)^{p}$ on $|z| \leqslant$ $r_{k} / R_{k}$. Using (1), (2), and the corona theorem for $\Delta$, we can choose $g_{i, k} \in H^{\infty}(\Delta)$ uniformly bounded with $\sum_{i} f_{i, k} g_{i, k}=1, k=1,2, \ldots$ Let

$$
g_{1}=\sum\left(h_{k} g_{i, k}\right) \circ T_{k}-\sum\left(h_{k} g_{i, k}\right)\left(T_{k}(\lambda)\right)
$$


and $k=\sum f_{i} g_{i}$. By (1) and (3), the series for $g_{i}$ converges and defines an element of $H^{\infty}(\Delta)$. Now $\left|h^{p}-k\right| \rightarrow 0$ as $z \rightarrow \lambda$ and $m(k)=\sum m\left(f_{i}\right) m\left(g_{i}\right)=0$. This implies $m(h)^{p}=m\left(h^{p}\right)=m(k)=0$ and, hence, $m(h)=0$.

When $p=1$, the conclusion of Theorem 4.11 can easily be improved. The following corollary is a theorem of [4].

COROLlaRY 4.12. Let $\mathscr{D}=\mathscr{E} \backslash \cup \Delta_{n}$ where there are pairwise disjoint disks $D_{n}$ contained in $\mathscr{E}$ with center $\Delta_{n}=$ center $D_{n}$ and $\Sigma\left(\operatorname{rad} \Delta_{n} / \operatorname{rad} D_{n}\right)<\infty$. If the corona theorem holds for $\mathscr{E}$ then the corona theorem holds for $\mathscr{D}$, i.e. if $\mathscr{E}$ is dense in $\mathscr{M}(\mathscr{E})$, then $\mathscr{D}$ is dense in $\mathscr{M}(\mathscr{D})$.

Proof. Taking the sum of the Cauchy integrals around the $\Delta_{n}$ shows that $H^{\infty}(\mathscr{D}) \mid$ $\mathscr{M}_{\lambda}(\mathscr{D})$ is the direct sum of $H^{\infty}(\mathscr{E}) \mid \mathscr{M}_{\lambda}(\mathscr{D})$ and $I_{\lambda} \mid \mathscr{M}_{\lambda}(\mathscr{D})$. If $m \in \mathscr{M}(\mathscr{D})$ is not in the closure of $\mathscr{D}$, then it vanishes on $I_{\lambda}$ by Theorem 4.11. Hence $m$ is completely determined by its restriction $m_{0}$ to $H^{\infty}(\mathscr{E}), m_{0}=m \mid H^{\infty}(\mathscr{E})$. There is a net $\left\{z_{\lambda}\right\}$ in $\mathscr{E}$ which converges to $m_{0}$. We may assume $\lim _{n}\left(\operatorname{rad} D_{n} / d\left(c_{n}, \partial \mathscr{E}\right)\right)=0$ and so may choose $\left\{z_{\lambda}\right\}$ such that $z_{\lambda} \in \mathscr{E} \backslash \bigcup D_{n}$. In this case $\lim _{\lambda} f\left(z_{\lambda}\right)=0$ for all $f \in I_{\lambda}$ and $\left\{z_{\lambda}\right\}$ converges to $m$ in $\mathscr{M}(\mathscr{D})$, a contradiction.

When $\mathscr{D}=(\Delta \backslash\{0\}) \backslash \cup \Delta_{n}$, where the $\Delta_{n}$ have centers on the positive real axis which accumulate at 0 and $\sum\left(r_{n} / c_{n}\right)^{p}<\infty$ with $p \geqslant 2$, the corona theorem has been proved by J. Garnett (unpublished) using methods due to Hormänder. We remark that Theorem 4.10 in this case reduces the corona problem to the following question: If $\Phi(f)=\lim _{r \uparrow 0} f(r)$ whenever this limit exists, must $\Phi$ be contained in the closure of the negative axis in $\mathscr{M}(\mathscr{D})$ ?

We are finally ready for the big theorem.

THEOREM 4.13. Each nontrivial Gleason part in $\mathscr{T}$ is an analytic disk. A point in $\mathscr{T}$ lies in a nontrivial Gleason part if and only if it is contained in the closure of an interpolating sequence.

Proof. The function $\sum_{N}^{\infty}\left(z^{p} \circ T_{n}\right)-\sum_{N}^{\infty} z^{p} \circ T_{n}(\lambda)$ has modulus 1 on $\mathscr{T} \cap \mathscr{M}_{\lambda}(\mathscr{D})$, has modulus less than 1 on $\mathscr{M}_{\lambda}(\mathscr{D}) \backslash \mathscr{T}$ (this is certainly true for points in the closure of $\mathscr{D}$ and, by Theorem 4.11 , any homomorphisms in $\mathscr{M}_{\lambda}(\mathscr{D})$ not in the closure of $\mathscr{D}$ contain this function in their kernel), and can be chosen to have norm less than $1+\varepsilon$ and to be less than $\varepsilon$ in modulus outside of a given neighborhood of $\lambda$. It follows that the Gleason part of a point in $\mathscr{T} \cap \mathscr{M}_{\lambda}(\mathscr{D})$ is entirely contained in $\mathscr{T} \cap \mathscr{M}_{\lambda}(\mathscr{D})$.

If $m, n \in \mathscr{T} \cap \mathscr{M}_{\lambda}(\mathscr{D})$, with $n \neq m$, then there is an $f \in H^{\infty}(\mathscr{D})$, with $\|f\| \leqslant 1$, $\hat{f}(m)=0$ and $\hat{f}(n) \neq 0$. If $m$ is not in the closure of an interpolating sequence, then by Theorem 4.9 and Lemma 4.10 , we may find $g, h \in H^{\infty}(\mathscr{D})$ such that $\|g\|$, $\|h\| \leqslant 1+\varepsilon, \hat{g}(m)=\hat{h}(m)=0$ and $|\hat{g}(n)||\hat{h}(n)|=|\hat{f}(n)|$. If the function $f$ satisfies

$$
|\hat{f}(n)|>p(m, n)^{2}=\sup \left\{|\hat{k}(n)|: k \in H^{x}(\mathscr{D}),\|k\| \leqslant 1, \hat{k}(m)=0\right\}^{2},
$$

then if, say, $|\hat{g}(n)| \geqslant|\hat{h}(n)|$, then

$$
\left\|\frac{g}{1+\varepsilon}\right\| \leqslant 1, \quad \frac{\hat{g}}{1+\varepsilon}(m)=0 \text { and } \quad\left|\frac{\hat{g}}{1+\varepsilon}(n)\right| \geqslant \frac{1}{1+\varepsilon} \sqrt{\hat{f}(n)} .
$$


If $\varepsilon$ is sufficiently small, this last term is greater than $p(m, n)$, which is impossible. Hence $p(m, n)^{2}$ is not less than $p(m, n)$, i.e. $p(m, n)=1$ and $\{m\}$ is a point part.

The remainder of the theorem is contained in Theorem 4.10 .

Note that Theorem 4.11 is weaker than the corresponding theorem in Hoffman [7]. Hoffman proves that in the disk, if a maximal ideal is not contained in the closure of an interpolating sequence, then it is equal to its own square (in the strongest sense possible). Since our factorization is only good for the modulus on $\mathscr{T} \cap \mathscr{M}_{\lambda}(\mathscr{D})$, we obtain no information on this stronger assertion.

In the unit disk every bounded harmonic function has a continuous extension to $\mathscr{M}(\Delta)$. A similar result holds for $\mathscr{T}$.

THEOREM 4.14. Every bounded harmonic function defined on $\mathscr{D}$ has a continuous extension to $\mathscr{D} \cup \mathscr{T}$.

Proof. Let $h$ be a bounded real valued harmonic function defined on $\mathscr{D}$. Let $h_{n}$ be the harmonic extension to $\Delta$ of the boundary value of $h \circ T_{n}^{-1}$ on $\partial \Delta$. Let $g_{n}$ be a harmonic conjugate of $h_{n}$. Let $\lambda \in \partial \mathscr{D}$ and let

$$
f=\sum\left(z^{p} e^{h_{n}+i g_{n}}\right) \circ T_{n}-\sum\left(z^{p} e^{h_{n}+i g_{n}}\right)\left(T_{n}(\lambda)\right) .
$$

Then given $\varepsilon>0$ there is a $\delta>0$ and an $M$ such that for any $\delta^{\prime}<\delta,|h-\log | f||<\varepsilon$ for all $z$ satisfying $|z-\lambda|<\delta^{\prime}$ and $z \in \mathscr{D} \backslash \bigcup\left\{S\left(c_{n}, R r_{n}\right):\left|c_{n}-\lambda\right|<\delta^{\prime}\right\}$. The function $\log |f|$ thus provides a continuous extension of $h$ to $\mathscr{M}_{\lambda}(\mathscr{D}) \cap \mathscr{T}$. Since $\sum\left(z^{p} e^{h_{n}+i g_{n}}\right)\left(T_{n}(\lambda)\right)$ varies continuously with $\lambda$ on $\partial \mathscr{D}$, this extension is actually continuous on $\mathscr{D} \cup \mathscr{T}$.

5. Nontangential Gleason parts. In the open unit disk, associated with radial approach to the boundary is the concept of nontangential approach to the boundary. The sequence $\alpha_{n}$ approaches 1 nontangentially if $\lim \alpha_{n}=1$ and $\sup _{n} \inf _{z \in\{0,1)} \psi_{0}\left(z, \alpha_{n}\right)<\infty$. We will say that a sequence in $\mathscr{D}=(\Delta \backslash\{0\}) \backslash \cup \Delta_{n}$ approaches $0 \pi$-nontangentially if $\lim \alpha_{n}=0$ and $\sup _{n} \inf _{z \in[-1 / 2.0)} \pi\left(z, \alpha_{n}\right)<\infty$. A homomorphism in $\mathscr{M}_{0}(\mathscr{D})$ is said to be a nontangential homomorphism if it is contained in the closure of a sequence in $\mathscr{D}$ which converges to $0 \pi$-nontangentially. A homomorphism in $\mathscr{M}_{0}(\mathscr{D})$ which is not nontangential is tangential.

In the remainder of this section $\mathscr{D}$ will always be an $L$-domain satisfying $\sup \left(c_{n+1} / c_{n}\right)<1$ and $\sum\left(r_{n} / c_{n}\right)^{p}<\infty$, some integer $p \geqslant 1$. For such domains a sequence $\left\{\alpha_{n}\right\}$ converges $\pi$-nontangentially to 0 if and only if it converges to 0 and $\left\{\alpha_{n}\right\} \subset \mathscr{D} \backslash \cup S\left(c_{n}, R r_{n}\right)$ for some $R>1$ (cf. Lemma 3.5). Let $T_{n}(z)=r_{n} /\left(z-c_{n}\right)$ and $U_{n}(z)=c_{n}+r_{n} / z$. Let $\omega \in \beta \backslash N, n_{\lambda} \rightarrow \omega$, and $-c_{n_{\lambda}}$ converge to $m \in$ $\left[\frac{-1}{2}, 0\right)^{-\mathscr{M}(D)}$ for $[0,1)$.

THEOREM 5.1. 1. For each $f \in H^{\infty}(\mathscr{D}), f \circ U_{n_{\lambda}}$ converges uniformly on compact subsets of $\Delta \backslash\{0\}$ to an analytic function defined on $\Delta \backslash\{0\}$.

Let $U_{\omega}$ be the resulting mapping of $\Delta \backslash\{0\}$ into $\mathscr{M}(\mathscr{D})$ defined by $U_{\omega}(z)(f)=$ $\lim f \circ U_{n_{\lambda}}(z)$.

2. $U_{\omega}$ is an injective analytic mapping of $\Delta \backslash\{0\}$ into the set of nontangential homomorphisms in $\mathscr{M}_{0}(\mathscr{D})$. 
3. $U_{\omega}(\Delta \backslash\{0\}) \cap U_{\omega^{\prime}}(\Delta \backslash\{0\})=\varnothing$ if $\omega \neq \omega^{\prime}$.

4. The set of nontangential homomorphisms in $\mathscr{M}_{0}(\mathscr{D})$ is equal to the disjoint union of $\left[\frac{-1}{2}, 0\right)^{-M(D)} \backslash\left[\frac{-1}{2}, 0\right)$ and $U\left\{U_{\omega}(\Delta \backslash\{0\}): \omega \in \beta N \backslash N\right\}$.

5. If $-c_{n_{\lambda}}$ converges to $m \in\left[\frac{-1}{2}, 0\right)^{-M(D)}$, where $n_{\lambda} \rightarrow \omega$, then $\lim _{z \rightarrow 0} \hat{f} \circ U_{\omega}(z)=$ $\hat{f}(m)$ for all $f \in H^{\infty}(\mathscr{D})$.

Proof. 1. $U_{n_{\lambda}}^{-1}(\mathscr{D})$ eventually contains each compact subset of $\Delta \backslash\{0\}$ and it is automatic that $f \circ U_{n_{\lambda}}(z)$ converges for each $z \in \Delta \backslash\{0\}$ and $f \in H^{\infty}(\mathscr{D})$. Pick's theorem implies that $f \circ U_{n_{\lambda}}$ converges uniformly on a neighborhood of each point in $\Delta \backslash\{0\}$ and hence uniformly on compact subsets of $\Delta \backslash\{0\}$. The limit function is automatically analytic and defined on $\Delta \backslash\{0\}$.

2. Let

$$
f=\sum\left(\frac{r_{n}}{z-c_{n}}\right)^{p}-\sum\left(\frac{r_{n}}{-c_{n}}\right)^{p} \text { and } g=\sum\left(\frac{r_{n}}{z-c_{n}}\right)^{p+1}-\sum\left(\frac{r_{n}}{-c_{n}}\right)^{p+1} \text {. }
$$

Then $\hat{f} \circ U_{\omega}=z^{p}$ and $\hat{g} \circ U_{\omega}=z^{p+1}$, so $U_{\omega}$ is injective. It is analytic by (1). Elementary estimates on the Cauchy integral show that if $\alpha_{n} \rightarrow 0, R>1$, and $\left\{\alpha_{n}\right\} \subset$ $\mathscr{D} \backslash \cup \Delta\left(c_{n}, R r_{n}\right)$, then $\lim \sup \pi\left(\left[\frac{-1}{2}, 0\right), \alpha_{n}\right) \leqslant \log (R+1 / R-1)$ (cf. Lemma 3.5), so $U_{\omega}(\Delta \backslash\{0\})$ contains only nontangential homomorphisms.

3. If $\omega \neq \omega^{\prime}$, then there is a set $S \subset N$ with $\omega \in S^{-\beta N}, \omega^{\prime} \notin S^{-\beta N}$. Let

$$
f_{S}=\sum_{n \in S}\left(\frac{r_{n}}{z-c_{n}}\right)^{p}-\sum_{n \in S}\left(\frac{r_{n}}{-c_{n}}\right)^{p}
$$

Then $\hat{f}_{S} \circ U_{\omega}=z^{p}$ and $\hat{f}_{S} \circ U_{\omega^{\prime}}=0$.

4. The function $f$ of (1) satisfies $\hat{f}=0$ on $\left[\frac{-1}{2}, 0\right)^{-\mathscr{M}(\mathscr{D})} \backslash\left[\frac{-1}{2}, 0\right), 0<|\hat{f}|<1$ on $\cup\left\{U_{\omega}(\Delta \backslash\{0\}): \omega \in \beta N \backslash N\right\}$, and $|\hat{f}|<1$ on the set of nontangential homomorphisms at 0 .

5. As in (2), $\lim \sup \pi\left(-c_{n}, c_{n}+R r_{n}\right) \leqslant \log (R+1 / R-1)$, so $\pi\left(m, U_{\omega}(1 / R)\right) \leqslant$ $\log (R+1 / R-1)$.

COROllaRy 5.2. Every nontangential homomorphism in $\mathscr{M}_{0}(\mathscr{D})$ is contained in an analytic disk.

We can describe the nontangential Gleason parts in greater detail. In the following theorem we will assume the corona theorem holds (as Garnett has proved). Alternately, we may take "Gleason part" to mean that portion of the Gleason part which lies in the closure of $\mathscr{D}$.

Define a mapping $\Psi: N \rightarrow \mathscr{D}$ by $\Psi(n)=-c_{n}$. Let $\tilde{\Psi}: \beta N \rightarrow \mathscr{M}(\mathscr{D})$ be the continuous extension of $\Psi$ to $\beta N$. Let $g(z)=\int_{E} d x /(x-z)$ for $z \in\left[\frac{-1}{2}, 0\right)$ and let $\tilde{g}:\left[\frac{-1}{2}, 0\right)^{-\mathscr{M}(\mathscr{D})} \rightarrow \mathscr{E}$ as in $\S 2$. Let $W: \mathscr{E} \rightarrow \mathscr{M}(\mathscr{E})$ be a Wermer map in the closure of the mappings $z \rightarrow z+a, a \in R^{+}$. The function $\tilde{g} \circ(W \mid R)$ is an injective continuous mapping of $R$ onto the intersection of $\mathscr{G}(W(0))$ with [ $\left.\frac{-1}{2}, 0\right)^{-\mathscr{M}(\mathscr{D})}$ (Theorem 2.9).

THEOREM 5.3. In the space $R \cup \tilde{\Psi}^{-1}\left(\tilde{g}^{-1}(W(R)) \times \Delta\right)$ identify each set $\tilde{\Psi}^{-1}\left(\tilde{g}^{-1}(W(r)) \times\{0\}\right) \cup\{r\}$ to the point $r$ to form a space $\chi$. The Gleason part containing $\tilde{g}^{-1}(W(0))$ is a continuous bijective image of $\chi$ under a mapping under which 
each Gelfand transform $\hat{f}, f \in H^{\infty}(\mathscr{D})$ goes into a function which is "analytic" on each set of the form $\{\omega\} \times \Delta, \omega \in \beta N$.

Proof. Define a mapping $\Phi$ from $R \cup\left\{\tilde{\Psi}^{-1}\left(\tilde{g}^{-1}(W(R)) \times \Delta\right)\right\}$ into $\mathscr{M}(\mathscr{D})$ as follows: Let $r \in R, \quad w \in \tilde{\Psi}^{-1}\left(\tilde{g}^{-1}(W(r))\right)$, and $z \in \Delta$. Let $\Phi(r)=$ $\left.\tilde{g}^{-1}(W(r)), \Phi(\omega, z)\right)=U_{\omega}(z)$ if $z \neq 0$, and $\Phi(\omega, 0)=\tilde{g}^{-1}(W(r))$. Let $f \in H^{\propto}(\mathscr{D})$ and $\varepsilon>0$. If $\hat{f}(\Phi(\omega, \mathrm{z}))=a$, some $z \neq 0$, then by Pick's theorem and the definition of $U_{\omega}, A^{-\beta N} \times S(z, \delta) \subset \hat{f}^{-1}(S(a, \varepsilon))$ for some $\delta>0$, and $\omega \in A^{-\beta N}$. The mapping $\Phi$ is thus continuous on $\tilde{\Psi}^{-1}(W(R)) \times(\Delta \backslash\{0\})$. By the definition of the metric $\pi$,

$$
\tilde{\Psi}^{-1}\left(\tilde{g}^{-1}(W(r-\varepsilon / 8, r+\varepsilon / 8))\right) \times S(0, \varepsilon / 2) \subset \hat{f}^{-1}(S(a, \varepsilon))
$$

if $\|f\| \leqslant 1$ and $\hat{f}(\Phi(\omega, 0))=a$. Hence $\Phi$ is continuous on each point of $\tilde{\Psi}^{-1}\left(\tilde{g}^{-1}(W(R))\right) \times\{0\}$. That $\Phi$ is continuous on $R$ is Theorem 2.9. Now $\Phi$ is constant on each set $\tilde{\Psi}^{-1}\left(\tilde{g}^{-1}(W(r)) \times\{0\}\right) \cup\{r\}$, and if we identify these sets to points, the resulting mapping $\tilde{\Phi}$ is continuous and bijective.

Gathering together results we have already proved, we may state the following characterization of nontrivial Gleason parts for the domains we are studying.

THEOREM 5.4. Let $\mathscr{D}=(\Delta \backslash\{0\}) \backslash U \Delta_{n}$ with $\sup \left(c_{n+1} / c_{n}\right)<1$ and $\sum\left(r_{n} / c_{n}\right)^{p}<$ $\infty, p \geqslant 1$. If $p \geqslant 2$, the following are equivalent $(m \in \mathscr{M}(\mathscr{D}))$.

(a) The Gleason part containing $m$ is nontrivial.

(b) $m$ is contained in the closure of an interpolating sequence.

(c) $m$ is contained in an analytic set.

(d) A neighborhood basis at $m$ is given by the sets $S_{\pi}(S, \varepsilon)^{-\mathscr{M}(\mathscr{D})}$ where $\varepsilon>0$ and $S$ varies over all subsets of $\mathscr{D}$ satisfying $m \in S^{-\mathscr{M}(\mathscr{D})}$.

(e) If $A$ and $B$ are $\pi$-separated from each other, then $m \notin A^{-\mathscr{M}(\mathscr{D})} \cap B^{-\mathscr{M}(\mathscr{D})}$.

(f) The following is false: If $A \subset \mathscr{D}$ with $\sup _{z \in \mathscr{D}} \pi(z, A)<\infty$, then $m \in A^{-\mathscr{M}(\mathscr{D})}$.

If $p=1$, conditions (a)-(f) are equivalent except at the distinguished homomorphism and:

(g) The distinguished homomorphism is not contained in an interpolating sequence.

(h) The distinguished homomorphism is contained in an analytic set.

(i) A neighborhood basis for the distinguished homomorphism is given by $S_{\pi}\left(z_{n}, 1 / n\right)^{-\mathscr{M}(\mathscr{D})}$ for some sequence $\left\{z_{n}\right\} \subset \mathscr{D}$ with $\lim z_{n}=0$.

Proof. We have already seen the equivalence of (a), (b), and (c). If $S \subset \mathscr{D}$ is interpolating and $m \in S^{-\mathscr{M}(\mathscr{D})}$, it is a consequence of Lemma 4.5 that $\left\{S(T, \varepsilon)^{-\mathscr{M}(\mathscr{D})}\right.$ : $\left.T \subset S, m \in T^{-\mathscr{M}(\mathscr{D})}, \varepsilon>0\right\}$ is a neighborhood base at $m$, so (b) $\Rightarrow(\mathrm{d})$. Suppose (e) is false. Let $A, B \subset \mathscr{D}$ be $\pi$-separated from each other, with $\pi(A, B)=\varepsilon>0$ and $m \in A^{-\mathscr{M}(D)} \cap B^{-\mathscr{M}(\mathscr{D})}$. Then $S(A, \varepsilon / 2)^{-\mathscr{M}(\mathscr{D})}$ is not a neighborhood of $m$ since $\mathscr{M}(\mathscr{D}) \backslash S(A, \varepsilon / 2)^{-\mathscr{M}(\mathscr{D})} \supset B$ and $m \in B^{-\mathscr{M}(\mathscr{D})}$. Hence $(\mathrm{d}) \Rightarrow(\mathrm{e})$.

Suppose (f) is false, and let $S \subset \mathscr{D}$ be a set with $\sup _{z \in \mathscr{D}} \pi(z, S)<\infty$. The set $S$ can be written as a union $S=A_{1} \cup A_{2}$ such that $A_{1}$ and $A_{2}$ are $\pi$-separated from each other and $\sup _{: \in \mathscr{D}} \pi\left(z, A_{i}\right)<\infty$ for $i=1,2$. Then $m \in A_{1}^{-\mathscr{M}(\mathscr{D})} \cap A_{2}^{-\mathscr{M}(\mathscr{D})}$. Hence $(e) \Rightarrow$ (f).

If $\{m\}$ is a point part and $\lim z_{\lambda}=m$, then the mappings $F_{\lambda}$ of Theorem 4.6 converge to a constant map $F_{m}$ defined by $\hat{f} \circ F_{m}(z)=m(f)$ for all $f \in H^{\infty}(\mathscr{D})$, 
$z \in \Delta$. If $A \subset \mathscr{D}$ with $\sup _{z \in \mathscr{D}} \pi(z, A)<\infty$ and $w_{\lambda}$ minimizes $\left\{\pi\left(z_{\lambda}, w\right): w \in A\right\}$, then $\sup _{\lambda} \pi\left(z_{\lambda}, w_{\lambda}\right)<\infty$. Hence $\sup _{\lambda}\left|F_{\lambda}^{-1}\left(w_{\lambda}\right)\right|<1$ and $\lim f\left(w_{\lambda}\right)=m(f)$ for all $f \in H^{\infty}(\mathscr{D})$, i.e. $m \in A^{-\mathscr{M}(\mathscr{D})}$. Thus (f) $\Rightarrow$ (a).

Statements $(\mathrm{g})$ and $(\mathrm{h})$ have been discussed and (i) is an immediate consequence of (d).

In the disk each bounded harmonic function has a continuous extension to $\mathscr{M}(\Delta)$. In more general domains this may not be the case.

THEOREM 5.5. Let $\mathscr{D}=(\Delta \backslash\{0\}) \backslash \cup \Delta_{n}$ with $\sup \left(c_{n+1} / c_{n}\right)<1$ and $\sum\left(r_{n} / c_{n}\right)^{p}<$ $\infty$, some $p \geqslant 1$. Every bounded harmonic function on $\mathscr{D}$ has a continuous extension to (the closure of $\mathscr{D}$ in $) \mathscr{M}(\mathscr{D})$ if and only if $\sum\left(\log c_{n} / \log r_{n}\right)=\infty$.

Proof. If $\Sigma\left(\log c_{n} / \log r_{n}\right)<\infty$, then, by Theorem 3.8 , there is a sequence in $\left[\frac{-1}{2}, 0\right)$ which is not $\pi$-separated but interpolates bounded harmonic functions on $\mathscr{D}$. There is then a bounded harmonic function on this sequence interpolating zeros and ones which does not have a continuous extension to $\mathscr{M}(\mathscr{D})$.

If $\sum\left(\log c_{n} / \log r_{n}\right)=\infty$, then 0 is a regular point for the Dirichlet problem on $\mathscr{D}$ and every bounded harmonic function on $\mathscr{D}$ has a limit as $r$ goes to 0 along the negative axis (see Theorem 3.7). This, together with Theorem 4.14, proves the theorem.

A bounded function on $\mathscr{D}$ is said to be $\pi$-normal if it is uniformly continuous when $\mathscr{D}$ is equipped with the Carathéodory metric.

THEOREM 5.6. Let $\mathscr{D}=(\Delta \backslash\{0\}) \backslash \bigcup \Delta_{n}$ with $\sup \left(c_{n+1} / c_{n}\right)<1$ and $\sum\left(r_{n} / c_{n}\right)^{p}<$ $\infty$, some $p \geqslant 1$. A bounded harmonic function defined on $\mathscr{D}$ has a continuous extension to $\mathscr{M}(\mathscr{D})$ if and only if it is $\pi$-normal.

Proof. Any bounded function which has a continuous extension to $\mathscr{M}(\mathscr{D})$ is $\pi$-normal. If $\left\{\alpha_{n}\right\},\left\{\beta_{n}\right\} \subset \mathscr{D}$, with $\lim \pi\left(\alpha_{n}, \beta_{n}\right)=0$ and inf $\left|f\left(\alpha_{n}\right)-f\left(\beta_{n}\right)\right| \geqslant \delta>$ 0 , and $m \in\left\{\alpha_{n}\right\}^{-\mathscr{M}(\mathscr{D})} \backslash \mathscr{D}$, then also $m \in\left\{\beta_{n}\right\}^{-\mathscr{M}(\mathscr{D})}$, so $f$ cannot have a continuous extension to $\mathscr{D} \cup\{m\}$. Using Theorem 4.14 it suffices for the converse to show that a bounded $\pi$-normal harmonic function has a continuous extension to $\left[\frac{-1}{2}, 0\right)^{-\mathscr{M}(\mathscr{D})}$.

Actually it is true that any $\pi$-normal function defined on $\mathscr{D}$ has a continuous extension to the set of nontangential homeomorphisms at 0 . This is an immediate consequence of Corollary 5.2 and Theorem 5.4. Let $f$ be $\pi$-normal on $\mathscr{D}$. Let $m$ be a nontangential homeomorphism and let $S_{1}, S_{2} \subset \mathscr{D}$ with $m \in S_{1}^{-\mathscr{M}(\mathscr{D})}$ and $m \in$ $S_{2}^{-\mu(D)}$. Then $S_{1}$ and $S_{2}$ cannot be $\pi$-separated so $\overline{f\left(S_{1}\right)} \cap \overline{f\left(S_{2}\right)} \neq \varnothing$. Let $a \in$ $\bigcap\left\{\overline{f(S)}: S \subset \mathscr{D}\right.$ and $\left.m \in S^{-\mathscr{M}(\mathscr{D})}\right\}$. For $\varepsilon>0, A=A(a, \varepsilon)=\{z \in \mathscr{D}:|f(z)-a|<$ $\varepsilon / 2\}$ is nonempty. If $\delta>0$ is such that $\pi(z, w)<\delta$ and $|z|,|w| \leqslant 2|a| \Rightarrow \mid f(z)-$ $f(w) \mid<\varepsilon / 2$, then $S(A, \delta)^{-\mathscr{M}(\mathscr{D})} \subset f^{-1}(S(a, \varepsilon))$. Then $m(f)=\bigcap\{\overline{f(S)}: S \subset \mathscr{D}$ and $\left.m \in S^{-\mathscr{M}(\mathscr{D})}\right\}$ defines a continuous extension of $f$ to the set of nontangential homomorphisms.

6. Nonstandard analysis. In a nonstandard model the domain $\mathscr{D}$ has an extension $\mathscr{D}^{*}$. Each $f \in H^{\infty}(\mathscr{D})$ has an extension $f^{*}$ that maps $\mathscr{D}^{*}$ into $\mathbf{C}^{*}$. There is a natural mapping of $\mathscr{D}^{*}$ into $\mathscr{M}(\mathscr{D})$ defined by $F(z)(f)={ }^{o}\left(f^{*}(z)\right), z \in \mathscr{D}^{*}, f \in H^{\infty}(\mathscr{D})$, 
where ${ }^{o}$ indicates the standard part. $F$ maps $\mathscr{D}^{*}$ onto $\mathscr{M}(\mathscr{D})$ if and only if the Corona Theorem holds for $\mathscr{D}$. There are three natural metrics on $\mathscr{D}$ which are useful for our purposes; the Poincaré metric $\psi, \sigma$ defined locally by $|d z| / d(z, \partial \mathscr{D})$, and the Carathéodory metric $\pi$. Each of these metrics extends to a nonstandard metric on $\mathscr{D}^{*}$. Being infinitesimally far apart or finitely far apart, in each of these metrics, defines an equivalence relation on $\mathscr{D}^{*}$. The resulting equivalence classes are the monads, $M(z)$, and galaxies, $G(z)$, of $\mathscr{D}^{*}$ in these metrics. For $z \in \mathscr{D}^{*}, M_{\sigma}(z)$ $\subset M_{\psi}(z) \subset M_{\pi}(z)$ and $G_{\sigma}(z) \subset G_{\psi}(z) \subset G_{\pi}(z)$. Each of these sets maps into a subset of $\mathscr{M}(\mathscr{D})$ under $F$. In the case when $\mathscr{D}=\Delta$, these three metrics are equivalent, so the galaxies and monads under these metrics are the same. The images of the galaxies under $F$ are precisely the Gleason parts of $\mathscr{M}(\Delta)$. When $\mathscr{D}$ is infinitely connected, the situation may be much more complicated. It can be shown that $F\left(G_{o}(z)\right)$ is always either a point or an analytic set, and the nature of this analytic set can be determined from the geometry of $\mathscr{D}$. The important idea is the "type" discussed in [2]. When the metric $\psi$ is not equivalent to $\sigma$, a new phenomena may occur. Analytic sets in $\mathscr{M}(\mathscr{D})$ which arise from the $\sigma$-galaxies in $\mathscr{D}^{*}$ may be "glued together" along isolated sets of points. This is one reason why in the domains considered in this paper and in [4], there occur many disks in $\mathscr{M}(\mathscr{D})$ with their origins identified. The $\pi$-galaxies may be very large. A $\pi$-galaxy is a union of o-galaxies and has an image under $F$ which is contained in a single Gleason part: $F\left(G_{\pi}(z)\right) \subset \mathscr{G}(F(z))$. In all known examples, equality occurs here. Even in the case of the disk this is nontrivial and is the "deep" part of Hoffman's paper [7]. The "hedges" discussed in this paper and the "congested gardens" of [4] are examples of Gleason parts arising from large $\pi$-galaxies. The general problem of describing the Gleason parts of $\mathscr{M}(\mathscr{D})$ has the following aspects: First, it is necessary to describe the possible structures that can occur in $\pi$-galaxies. In the disk this is trivial since $\pi$-galaxies are $\sigma$-galaxies, but in general this is a deep problem. To be more precise, for each $\pi$-galaxy $G_{\pi}(z)$, a metric space $\left(X_{\pi}(z), p\right)$ is formed from the $\pi$-monads contained in $G_{\pi}(z)$ by defining $p\left(M_{\pi}(n), M_{\pi}(v)\right)={ }^{o}(\pi(u, v))$. In the case $\mathscr{D}=\Delta$, $\left(X_{\pi}(x), p\right)$ is always isometric to $\left(\Delta, \psi_{0}\right)$. The problem is to describe the metric spaces $\left(X_{\pi}(z), p\right)$ that can occur.

There is a natural continuous mapping of $\left(X_{\pi}(z), p\right)$ into $\mathscr{M}(\mathscr{D})$ defined by $M_{\pi}(z) \rightarrow F(z)$. In the case $\mathscr{D}=\Delta$ this mapping is either constant or injective. In the $L$-domains considered in this paper, this mapping need not be injective or constant. In fact, the space $\left(X_{\pi}(z), p\right)$ corresponding to a "hedge" may have arbitrarily high cardinality, depending on the nonstandard model used. Having determined the nature of $\left(X_{\pi}(z), p\right)$, it is then necessary to determine how this space is "collapsed" to form a set in $\mathscr{M}(\mathscr{D})$. In the nonstandard language, $\left(X_{\pi}(z), p\right)$ is determined by all the finite-valued internal analytic functions on $\mathscr{D}^{*}$, and the image of this set in $\mathscr{M}(\mathscr{D})$ is determined by all standard analytic functions on $\mathscr{D}^{*}$. Since the question of the existence of internal and standard objects may involve quite different sorts of problems, this division of the general problem of describing the Gleason parts of $\mathscr{M}(\mathscr{D})$ into the problems of describing the metric spaces $\left(X_{\pi}(z), p\right)$ and then describing how these spaces are collapsed to form sets in $\mathscr{M}(\mathscr{D})$ is of fundamental importance. 
It is also necessary to show that whole Gleason parts are obtained in this way, i.e. to show that $F\left(G_{\pi}(z)\right)=\mathscr{G}(F(z))$.

In [2] the theory of the $\sigma$ - and $\psi$-galaxies of $\mathscr{D}^{*}$ is developed and examples are described which include the domains considered in this paper. [1] contains a general discussion of these ideas.

\section{REFERENCES}

1. M. Behrens, Analytic sets in $\mathscr{M}(\mathscr{D})$, Victoria Symposium on Nonstandard Analysis (Hurd and Loeb, eds.), Lecture Notes in Math., vol. 369, Springer-Verlag, Berlin, 1974.

2. __ Geometry of analytic sets in maximal ideal spaces (to appear).

3. The corona conjecture for a class of infinitely connected domains, Bull. Amer. Math. Soc. 76 (1970), 387-391.

4. . The maximal ideal space of algebras of bounded analytic functions on infinitely connected domains, Trans. Amer. Math. Soc. 161 (1971), 359-380.

5. J. Garnett, Interpolating sequences for bounded harmonic functions, Indiana Univ. Math. J. 21 (1971). No. 3, 187-192.

6. K. Hoffman, Banach spaces of analytic functions, Prentice-Hall, Englewood Cliffs, N. J., 1962.

7. Bounded analytic functions and Gleason parts, Ann. of Math. (2) 86 (1967), 74-111.

8. M. Tsuji, Potential theory, Maruzen. Tokyo, 1959.

9. L. Zalcman, Bounded analytic functions on domains of infinite connectivity, Trans. Amer. Math. Soc. 144 (1969), 241-270.

Department of Mathematics, University of California, Los Angeles, California 90024 